\title{
CROSS CORRELATION AND DECONVOLUTION OF NOISE SIGNALS IN RANDOMLY LAYERED MEDIA
}

\author{
JOSSELIN GARNIER* AND KNUT SøLNA ${ }^{\dagger}$
}

\begin{abstract}
It is known that cross correlation of waves generated by noise sources, propagating in an unknown medium, and recorded by a sensor array, can provide information about the medium. In this paper the medium is a three-dimensional small-scale randomly layered medium with slow macroscopic variations. The main objective is here to set forth a framework for analysis of cross correlations of waves generated by noise sources and propagating in such a medium, moreover, use this framework to design estimators for macroscale medium features. The noise sources are located at the bottom of a random medium slab and generate a random wave field that is scattered by the rapid random fluctuations of the medium and then recorded at the surface. Taking into account the pressure release boundary conditions at the surface, this situation corresponds to the so-called daylight configuration. The analysis is carried out in the asymptotic framework where the typical wavelength is small compared to the scale of the macroscopic variations of the background medium and large compared to the decoherence length of the random fluctuations of the medium. It is shown that the cross correlation of the waves recorded at the surface contains statistically stable information about the background medium.
\end{abstract}

Key words. Passive sensor imaging, noise sources, random media.

AMS subject classifications. 35R30, 35R60, 86A15.

1. Introduction. In this paper we consider background velocity estimation using noise sources in a geophysical context. We consider a medium (the earth's crust) that comprises two types of one-dimensional variations: slow, smooth, and deterministic variations that we want to image on the one hand, and rapid, possibly rough, and random fluctuations that are responsible for the main wave scattering on the other hand. One cannot hope to be able to, and typically one does not wish to, image the rapid fluctuations in the medium. However, we will show that slow variations in the medium can be imaged based on the deconvolution of correlations of wave fields recorded at the surface and emitted by noise sources in the subsurface.

We shall assume that acoustic noise sources located deep in the earth crust generate random stationary signals that propagate through the medium and are recorded at the surface. We study the properties of the cross correlation of the signals recorded at the surface with the specific objective of showing that it is possible to reconstruct the background properties of the medium, such as background velocity and dissipation. Travel time estimation and tomographic background velocity estimation using wave field cross correlations were studied by physicists $[12,13]$ and by geophysicists who have shown the efficiency of the method for travel time estimation $[6,14,16,18]$. More recently the Green's function estimation by cross correlation was analyzed by mathematicians in different frameworks (geometric optics [2, 4, 5, 10], parabolic approximation [7]). In contrast to these works, we consider in this paper a situation in which the random fluctuations of the medium play a very strong role and the observation region only consists of the surface of the random medium to be imaged. This is a typical model situation in the context of many inverse problems where only surface measurements are available.

* Laboratoire de Probabilités et Modèles Aléatoires \& Laboratoire Jacques-Louis Lions, Université Paris VII, 2 Place Jussieu, 75251 Paris Cedex 5, France garnier@math.jussieu.fr

${ }^{\dagger}$ Mathematics Department, University of California at Irvine, Irvine, CA 92697 ksolna@math.uci.edu 
Compared to reflection seismology there are no coherent source in our configuration. The sources are replaced by ambient or "internal" noise sources and we seek to exploit the response of these to infer knowledge about the medium, that is, to use the statistics of the transmitted wave field to solve an inverse problem. A main challenge is then to construct a functional of the measurements that gets out the "imprint" of the medium and to use this to solve an inverse problem. As mentioned, we will consider the case when the medium is layered and can then use the asymptotic theory developed in [8] for the analysis of wave propagation in randomly layered media. A main feature of our approach is that the imaging function we arrive at is motivated by this analytic framework and arises in a non-intuitive way. This is due to the fact that the recorded signals are multiply-scattered by the small-scale random fluctuations of the medium and therefore the imaging functional does not have a form which one could identify via formal reasoning based on travel time considerations. In [9] the problem of the identification of a source or a strong scatterer was considered in a similar setting, while we here look for information on the background medium where indeed there is no explicit or implicit travel time information that can be exploited.

The paper is organized as follows. In Section 2 we describe our model and introduce the cross correlation of the recorded signals. In Section 3 we study the case of a homogeneous medium and show that the depth of the sources can be estimated from the cross correlations. In Section 4 we describe the statistical properties of the generalized transmission coefficients of the medium (we here extend the results obtained in [8] to the case of pressure release boundary conditions). We use the statistical properties of the generalized transmission coefficients in Section 5 to describe the statistical properties of the cross correlation functions of the recorded signals. In Section 6 we show that it is possible to reconstruct the background medium from the cross correlation in a certain asymptotic regime. We present numerical simulations in Section 7.1 and apply our results (both theoretically and numerically) to the detection of an interface in Section 7.2. Our framework involves a number of different wave functionals and we summarize in Section 8 the general lines of the approach for medium inversion that we present in this paper.

2. Cross Correlations of Noise Signals. We consider linear acoustic waves propagating in a three-dimensional randomly layered medium. The governing equations are

$$
\rho^{\varepsilon}(z) \frac{\partial \overrightarrow{\boldsymbol{u}}}{\partial t}+\nabla p+\sigma^{\varepsilon}(z) \overrightarrow{\boldsymbol{u}}=\overrightarrow{\boldsymbol{F}}^{\varepsilon}(t, \boldsymbol{x}, z), \quad \frac{1}{K^{\varepsilon}(z)} \frac{\partial p}{\partial t}+\nabla \cdot \overrightarrow{\boldsymbol{u}}=0,
$$

where $p$ is the pressure field, $\overrightarrow{\boldsymbol{u}}$ is the three-dimensional velocity field, $\rho^{\varepsilon}$ is the density of the medium, $K^{\varepsilon}$ is the bulk modulus of the medium, $\sigma^{\varepsilon}$ is the dissipation, and $(\boldsymbol{x}, z) \in \mathbb{R}^{2} \times \mathbb{R}$ are the space coordinates. We consider in this paper the situation in which a randomly layered medium occupies the section $z \in(-L, 0)$. The medium $z \leq$ $-L$ is homogeneous and matched to the random section. Motivated by geophysical applications we assume that the density $\rho_{a}$ in the homogeneous half-space $z \geq 0$ is much smaller than the density $\rho(z)$ in the randomly layered medium for $z \leq 0$. Since the velocity and pressure are continuous away from the sources, the pressure in $z>0$ goes to zero and hence, by continuity, also at $z=0$. These are the so-called pressure release boundary conditions.

The sources are modeled by the forcing term $\overrightarrow{\boldsymbol{F}}^{\varepsilon}$. They are localized in the earth subsurface at $z=-L$, they impose a three-dimensional forcing, and they emit 


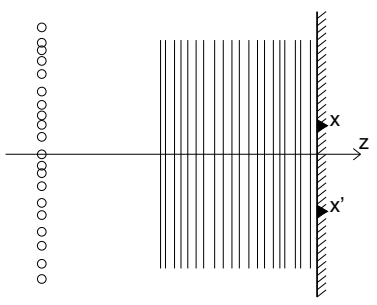

a)

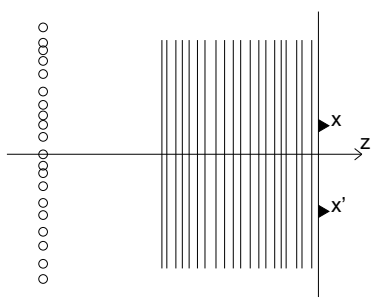

b)

FIG. 2.1. Acoustic daylight imaging configuration, with pressure release boundary conditions (a) and with matched (transparent) boundary conditions (b). The circles are the sources, the triangles are the sensors.

stationary random signals. We write the source term in the form

$$
\overrightarrow{\boldsymbol{F}}^{\varepsilon}(t, \boldsymbol{x}, z)=\overrightarrow{\boldsymbol{f}}^{\varepsilon}\left(\frac{t}{\varepsilon}, \frac{\boldsymbol{x}}{\varepsilon}\right) \delta(z-L),
$$

where $\boldsymbol{f}_{\boldsymbol{x}}^{\varepsilon}=\left(f_{x}^{\varepsilon}, f_{y}^{\varepsilon}\right)$ and $f_{z}^{\varepsilon}$ are zero-mean, time-stationary, and uncorrelated processes with the autocorrelation function

$$
\left\langle f_{x_{j}}^{\varepsilon}\left(\frac{t}{\varepsilon}, \frac{\boldsymbol{x}}{\varepsilon}\right) f_{x_{j}}^{\varepsilon}\left(\frac{t^{\prime}}{\varepsilon}, \frac{\boldsymbol{x}^{\prime}}{\varepsilon}\right)\right\rangle=\alpha_{x_{j}} F\left(\frac{t-t^{\prime}}{\varepsilon}\right) G\left(\frac{\boldsymbol{x}-\boldsymbol{x}^{\prime}}{\varepsilon}\right) \theta\left(\frac{\boldsymbol{x}+\boldsymbol{x}^{\prime}}{2}\right) .
$$

Here $\langle\cdot\rangle$ stands for the expectation with respect to the distribution of the noise sources and $\alpha_{x}=\alpha_{y}$ and $\alpha_{z}$ are nonnegative coefficients. The function $F$ is the local time covariance function, the function $G$ is the local spatial covariance function, and the nonnegative-valued function $\theta$ describes the spatial support of the source distribution. The functions $F$ and $G$ are assumed to be in $L^{1} \cap L^{\infty}$ and the function $\theta$ is assumed to be compactly supported and bounded. The Fourier transforms of $F$ and $G$ :

$$
\hat{F}(\omega)=\int F(t) e^{i \omega t} d t, \quad \hat{G}(\boldsymbol{k})=\int G(\boldsymbol{x}) e^{-i \boldsymbol{k} \cdot \boldsymbol{x}} d \boldsymbol{x},
$$

are nonnegative valued. Indeed the Fourier transform of the autocorrelation function of a stationary process is proportional to its power spectral density by Bochner's theorem. We assume, moreover, that $\hat{F}$ and $\hat{G}$ decay rapidly at infinity and that the functions $F$ and $G$ are normalized so that $F(0)=1$ and $\hat{G}(\mathbf{0})=1$. As is usual in geophysics and other applications the power spectral density is zero at zero-frequency: $\hat{F}(0)=0$. In this scaling, the decoherence time and length of the sources are of order $\varepsilon$. We remark here that in our setting the coherence time will play the role of a "typical" or "central wavelength". We shall thus consider an asymptotic setting corresponding to a high-frequency situation with the central wavelength being of order $\varepsilon$. However, we hasten to add that there will be an even finer scale in our model, namely the scale of variation of the random fluctuations and we describe these aspects of the model in more detail in Section 4, while we next describe the measurement configuration in more detail.

We observe the vertical velocity field $u^{\varepsilon}(t, \boldsymbol{x})$ at the surface $z=0$ during a (long) time interval of duration $T$. This is typical of seismographs, which usually record only the vertical velocity because it is less noisy and gives better records of some seismic waves. The set-up with pressure release boundary conditions at $z=0$, noise sources at $z=-L$, and velocity fields recorded at the surface $z=0$ is called acoustic daylight 
imaging by Claerbout [3] (Figure 2.1). He showed that the reflection response can be derived from the autocorrelation of the transmission response. The goal of our paper is to exploit the scattering properties of the medium and insight about how this affects wave statistics to estimate the (depth-dependent) background velocity, dissipation and scattering coefficient of the medium from the empirical cross correlation of the recorded signals defined by

$$
C_{T}^{\varepsilon}\left(t, \boldsymbol{x}, \boldsymbol{x}^{\prime}\right)=\frac{1}{T} \int_{0}^{T} u^{\varepsilon}\left(t^{\prime}, \boldsymbol{x}\right) u^{\varepsilon}\left(t^{\prime}+t, \boldsymbol{x}^{\prime}\right) d t^{\prime} .
$$

The following proposition (proved in [10]) shows that the empirical cross correlation is equivalent to the statistical cross correlation provided the recording time is large enough.

Proposition 2.1. The empirical cross correlation is a self-averaging quantity with respect to the distribution of the sources. We have

$$
C_{T}^{\varepsilon}\left(t, \boldsymbol{x}, \boldsymbol{x}^{\prime}\right) \stackrel{T \rightarrow \infty}{\longrightarrow} C^{\varepsilon}\left(t, \boldsymbol{x}, \boldsymbol{x}^{\prime}\right)
$$

in probability, where

$$
C^{\varepsilon}\left(t, \boldsymbol{x}, \boldsymbol{x}^{\prime}\right)=\left\langle u^{\varepsilon}(0, \boldsymbol{x}) u^{\varepsilon}\left(t, \boldsymbol{x}^{\prime}\right)\right\rangle .
$$

From the practical point of view, the experimental data consists of the empirical cross correlation $C_{T}^{\varepsilon}$, while the theoretical analysis of the paper is carried out with respect to the statistical cross correlation $C^{\varepsilon}$. These quantities are however equivalent assuming that $T$ is large enough.

3. The Recorded Field for a Dissipative Homogeneous Medium. In this section we assume that the density, bulk modulus, and dissipation of the medium are constant and denoted by $\rho_{0}, K_{0}$, and $\sigma_{0}$. The vertical velocity field recorded at the surface $z=0$ has the integral representation

$$
\begin{array}{r}
u^{\varepsilon}(t, \boldsymbol{x})=\frac{1}{(2 \pi)^{3}} \iint\left[\frac{\hat{f}_{z}^{\varepsilon}(\omega, \boldsymbol{\kappa})}{\zeta_{0}(\kappa)}+\frac{i \omega}{i \omega \rho_{0}-\varepsilon \sigma_{0}} \boldsymbol{\kappa} \cdot \hat{\boldsymbol{f}}_{\boldsymbol{x}}^{\varepsilon}(\omega, \boldsymbol{\kappa})\right] \exp \left(-i \frac{\omega}{\varepsilon}(t-\boldsymbol{\kappa} \cdot \boldsymbol{x})\right) \\
\times \exp \left(i \frac{\omega}{\varepsilon} \frac{L}{c_{0}(\kappa)}-\frac{\sigma_{0} c_{0}(\kappa)}{2 \zeta_{0} c_{0}} L\right) \omega^{2} d \boldsymbol{\kappa} d \omega
\end{array}
$$

The derivation involves a decomposition into plane wave modes that propagate undistorted in a homogeneous medium section, the use of jump conditions to identify their initial value at the source and the use of field continuity conditions to identify interface reflection coefficients at depths with jump in macroscale parameters. We shall explicitly go through this derivation in the more general case with random medium modulations in Appendix A. In (3.1) we have used the notation $\kappa=|\boldsymbol{\kappa}|$ and:

- $\hat{f}_{x_{j}}^{\varepsilon}(\omega, \boldsymbol{\kappa})$ is the Fourier transform of the random "source" function

$$
\hat{f}_{x_{j}}^{\varepsilon}(\omega, \boldsymbol{\kappa})=\iint f_{x_{j}}^{\varepsilon}(t, \boldsymbol{x}) e^{i \omega(t-\kappa \cdot \boldsymbol{x})} d t d \boldsymbol{x} .
$$

Its generalized covariance function is

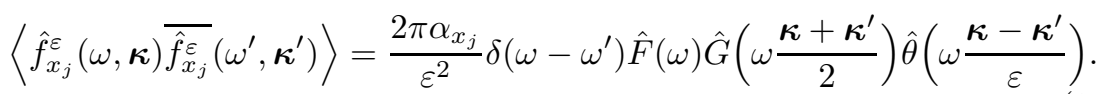


- $\zeta_{0}(\kappa)$ and $c_{0}(\kappa)$ are the mode-dependent impedance and velocity

$$
\zeta_{0}(\kappa)=\frac{\zeta_{0}}{\sqrt{1-\kappa^{2} c_{0}^{2}}}, \quad c_{0}(\kappa)=\frac{c_{0}}{\sqrt{1-\kappa^{2} c_{0}^{2}}} .
$$

We remark that the modes, as parameterized by the slowness vector $\boldsymbol{\kappa}$ and the frequency $\omega$, are evanescent if $\kappa>1 / c_{0}$.

- $\exp \left[i \omega L /\left(\varepsilon c_{0}(\kappa)\right)\right]$ gives the delay due to the mode-dependent vertical travel time $L / c_{0}(\kappa)$ from the source plane to the surface.

- $\exp \left[-\sigma_{0} c_{0}(\kappa) L /\left(2 \zeta_{0} c_{0}\right)\right]$ is the damping of the waves propagating from the source plane to the surface. Note that the damping depends on the mode and that there is a strong damping as the slowness magnitude approaches the evanescent regime.

Recall that we record the vertical velocity field at the surface and that we want to use its cross correlations in order the estimate macroscale features of the medium. We consider first the form of this cross correlation in the case when the medium is homogeneous (constant for $z<0$ ). This allows us to identify some principal relations in between the wave statistics and the medium parameters. For the cross correlation defined in (2.6) we have the following proposition.

PROPOSITION 3.1. The recorded field is a zero-mean stationary (in time) process whose cross correlation is given by:

$$
\begin{array}{r}
C^{\varepsilon}\left(-\varepsilon s, \boldsymbol{x}+\frac{\varepsilon \boldsymbol{y}}{2}, \boldsymbol{x}-\frac{\varepsilon \boldsymbol{y}}{2}\right)=\frac{1}{(2 \pi)^{5}} \iiint D^{\varepsilon}(\omega, \boldsymbol{\kappa}, \boldsymbol{\lambda}) \hat{F}(\omega) \hat{G}(\omega \boldsymbol{\kappa}) \hat{\theta}(\omega \boldsymbol{\lambda}) \\
\times \exp \left(i \frac{\omega}{\varepsilon}\left(\frac{L}{c_{0}\left(\left|\boldsymbol{\kappa}+\frac{\varepsilon \boldsymbol{\lambda}}{2}\right|\right)}-\frac{L}{c_{0}\left(\left|\boldsymbol{\kappa}-\frac{\varepsilon \boldsymbol{\lambda}}{2}\right|\right)}\right)-\frac{\sigma_{0} c_{0}\left(\left|\boldsymbol{\kappa}+\frac{\varepsilon \boldsymbol{\lambda}}{2}\right|\right)}{2 \zeta_{0} c_{0}} L-\frac{\sigma_{0} c_{0}\left(\left|\boldsymbol{\kappa}-\frac{\varepsilon \boldsymbol{\lambda}}{2}\right|\right)}{2 \zeta_{0} c_{0}} L\right) \\
\times \exp (-i \omega(s-\boldsymbol{\kappa} \cdot \boldsymbol{y}-\boldsymbol{\lambda} \cdot \boldsymbol{x})) \omega^{4} d \boldsymbol{\kappa} d \boldsymbol{\lambda} d \omega, \\
D^{\varepsilon}(\omega, \boldsymbol{\kappa}, \boldsymbol{\lambda})=\frac{\omega^{2}}{\rho_{0}^{2} \omega^{2}+\varepsilon^{2} \sigma_{0}^{2}}\left[\alpha_{x}\left(\kappa^{2}-\frac{\varepsilon^{2} \lambda^{2}}{4}\right)+\frac{\alpha_{z}}{\zeta_{0}\left(\left|\boldsymbol{\kappa}+\frac{\varepsilon \boldsymbol{\lambda}}{2}\right|\right) \zeta_{0}\left(\left|\boldsymbol{\kappa}-\frac{\varepsilon \boldsymbol{\lambda}}{2}\right|\right)}\right] .
\end{array}
$$

In the regime $\varepsilon \rightarrow 0$ we have

$$
\begin{aligned}
& C^{\varepsilon}\left(-\varepsilon s, \boldsymbol{x}+\frac{\varepsilon \boldsymbol{y}}{2}, \boldsymbol{x}-\frac{\varepsilon \boldsymbol{y}}{2}\right) \stackrel{\varepsilon \rightarrow 0}{\longrightarrow} \mathcal{C}(s, \boldsymbol{x}, \boldsymbol{y}), \\
& \begin{aligned}
\mathcal{C}(s, \boldsymbol{x}, \boldsymbol{y})= & \frac{1}{(2 \pi)^{3}} \iint D(\kappa) \hat{F}(\omega) \hat{G}(\omega \boldsymbol{\kappa}) \theta\left(\boldsymbol{x}-c_{0}(\kappa) \boldsymbol{\kappa} L\right) \\
& \times \exp \left(-\frac{\sigma_{0} c_{0}(\kappa)}{\zeta_{0} c_{0}} L-i \omega(s-\boldsymbol{\kappa} \cdot \boldsymbol{y})\right) \omega^{2} d \boldsymbol{\kappa} d \omega
\end{aligned} \\
& D(\kappa)=\frac{1}{\rho_{0}^{2}}\left[\frac{\alpha_{z}}{c_{0}^{2}}+\left(\alpha_{x}-\alpha_{z}\right) \kappa^{2}\right] .
\end{aligned}
$$

If the sources are isotropic $\alpha_{x}=\alpha_{z} \equiv \alpha$, then $D(\kappa)=\alpha / \zeta_{0}^{2}$. The proposition shows that the decoherence time and length of the recorded fields are of oder $\varepsilon$. This proposition moreover shows that accurate estimates of the depth $L$ of the sources and the background speed $c_{0}$ can be obtained. Indeed, this can be seen by the deconvolution step that we introduce next. We first introduce the Fourier transform:

$$
\widehat{\mathcal{C}}(\omega, \boldsymbol{\kappa}, \boldsymbol{\lambda}):=\iiint \mathcal{C}(s, \boldsymbol{x}, \boldsymbol{y}) \exp (i \omega(s-\boldsymbol{\kappa} \cdot \boldsymbol{y}-\boldsymbol{\lambda} \cdot \boldsymbol{x})) d s d \boldsymbol{y} d \boldsymbol{x},
$$


By (3.5) we find that:

$$
\widehat{\mathcal{C}}(\omega, \boldsymbol{\kappa}, \boldsymbol{\lambda})=D(\kappa) \hat{F}(\omega) \hat{G}(\omega \boldsymbol{\kappa}) \hat{\theta}(\omega \boldsymbol{\lambda}) \exp \left(-i \omega \boldsymbol{\lambda} \cdot \boldsymbol{\kappa} c_{0}(\kappa) L-\frac{\sigma_{0} c_{0}(\kappa)}{\zeta_{0} c_{0}} L\right) .
$$

Therefore, for $(\omega, \boldsymbol{\kappa}, \boldsymbol{\lambda})$ such that $\hat{F}(\omega) \hat{G}(\omega \boldsymbol{\kappa}) \hat{\theta}(\omega \boldsymbol{\lambda}) \neq 0$, the quantity

$$
\widehat{\mathcal{V}}(\omega, \boldsymbol{\kappa}, \boldsymbol{\lambda}):=\frac{\widehat{\mathcal{C}}(\omega, \boldsymbol{\kappa}, \boldsymbol{\lambda}) \widehat{\mathcal{C}}(\omega, \mathbf{0}, \mathbf{0})}{\widehat{\mathcal{C}}(\omega, \boldsymbol{\kappa}, \mathbf{0}) \widehat{\mathcal{C}}(\omega, \mathbf{0}, \boldsymbol{\lambda})}
$$

is well defined and its value is

$$
\widehat{\mathcal{V}}(\omega, \boldsymbol{\kappa}, \boldsymbol{\lambda})=\exp \left(-i \omega c_{0}(\kappa) \boldsymbol{\lambda} \cdot \boldsymbol{\kappa} L\right) .
$$

We can use this result to design the following algorithm to estimate the depth $L$ of the noise sources and the background speed $c_{0}$ :

1) compute the empirical cross correlation $C_{T}^{\varepsilon}$.

2) compute its Fourier transform $\widehat{C}_{T}^{\varepsilon}$ as in (3.6).

3) compute the quantity $\widehat{V}_{T}^{\varepsilon}$ as in (3.7):

$$
\widehat{V}_{T}^{\varepsilon}(\omega, \boldsymbol{\kappa}, \boldsymbol{\lambda}):=\frac{\widehat{C}_{T}^{\varepsilon}(\omega, \boldsymbol{\kappa}, \boldsymbol{\lambda}) \widehat{C}_{T}^{\varepsilon}(\omega, \mathbf{0}, \mathbf{0})}{\widehat{C}_{T}^{\varepsilon}(\omega, \boldsymbol{\kappa}, \mathbf{0}) \widehat{C}_{T}^{\varepsilon}(\omega, \mathbf{0}, \boldsymbol{\lambda})}
$$

4) by a least-square fit of the phase of the empirical function $\widehat{V}_{T}^{\varepsilon}(\omega, \boldsymbol{\kappa}, \boldsymbol{\lambda})$ with the theoretical function $-\boldsymbol{\omega} \boldsymbol{\lambda} \cdot \boldsymbol{\kappa} c_{0}(\kappa) L$ one can estimate the depth $L$ of the sources and the background speed $c_{0}$.

Note, however, that some special situations do not allow for the estimations of $L$ and $c_{0}$. In particular, if $\sigma_{0}=0$ and $\theta$ is constant, then (3.5) shows that the cross correlation does not depend on $L$ and $c_{0}$. The conditions under which the estimation is possible are discussed in Section 6.

We now proceed to consider the situation that is of principal interest to us, that is, when there are microscale and random fluctuations in the medium and how these provide additional possibilities for imaging.

4. The Recorded Field for a Dissipative Random Medium. We consider the general case with a random medium in the region $z \in(-L, 0)$. From now on the parameters of the medium in this region are assumed to be of the form

$$
\begin{aligned}
\left(K^{\varepsilon}\right)^{-1}(z) & = \begin{cases}K^{-1}(z) & \text { if } z \notin(-L, 0) \\
K^{-1}(z)\left[1+\nu\left(z, \frac{z}{\varepsilon^{2}}\right)\right] & \text { if } z \in(-L, 0)\end{cases} \\
\rho^{\varepsilon}(z) & =\rho(z) \\
\sigma^{\varepsilon}(z) & =\sigma(z) .
\end{aligned}
$$

The random process $\nu\left(z, z^{\prime}\right)$ models the random fluctuations in the medium. It is bounded in magnitude by a constant less than one, so that $K^{\varepsilon}$ is a positive quantity. For each $z$, the random process $z^{\prime} \mapsto \nu\left(z, z^{\prime}\right)$ is stationary and zero mean and it has strong mixing properties. The $z$-dependence of $\nu\left(z, z^{\prime}\right)$ models a depth-dependence of the statistical properties of the medium.

We define the background velocity and impedance by

$$
c(z)=\frac{\sqrt{K(z)}}{\sqrt{\rho(z)}}, \quad \zeta(z)=\sqrt{K(z) \rho(z)} .
$$


The background bulk modulus $K(z)$ and density $\rho(z)$ are smooth. This implies that the reflections induced by the macroscopic variations of $K, \rho$, and $\sigma$ are extremely small, they are dominated by incoherent reflections that are generated by the interaction of the waves with the small scale random fluctuations of the medium.

4.1. Integral Representation of the Recorded Field. We discuss now how the wave field, the vertical velocity field, at the surface can be described. The important generalization from the situation in the above section is that the wave field is defined in terms of a generalized transmission coefficient that captures the interaction of the wave with the random medium. The vertical velocity field recorded at the surface $z=0$ has the integral representation

$$
\begin{array}{r}
u^{\varepsilon}(t, \boldsymbol{x})=\frac{1}{(2 \pi)^{3}} \iint\left[\frac{\hat{f}_{z}^{\varepsilon}(\omega, \boldsymbol{\kappa})}{\zeta^{1 / 2}(\kappa,-L) \zeta^{1 / 2}(\kappa, 0)}+\frac{i \omega \boldsymbol{\kappa} \cdot \hat{\boldsymbol{f}}_{\boldsymbol{x}}^{\varepsilon}(\omega, \boldsymbol{\kappa})}{i \omega \rho(-L)-\varepsilon \sigma(-L)} \frac{\zeta^{1 / 2}(\kappa,-L)}{\zeta^{1 / 2}(\kappa, 0)}\right] \\
\times \mathcal{T}_{\omega, \kappa}^{\varepsilon} \exp \left(-i \frac{\omega}{\varepsilon}(t-\boldsymbol{\kappa} \cdot \boldsymbol{x})\right) \omega^{2} d \boldsymbol{\kappa} d \omega .
\end{array}
$$

Here:

- $\zeta(\kappa, z)$ and $c(\kappa, z)$ are the mode- and depth-dependent impedance and velocity

$$
\zeta(\kappa, z)=\frac{\zeta(z)}{\sqrt{1-\kappa^{2} c(z)^{2}}}, \quad c(\kappa, z)=\frac{c(z)}{\sqrt{1-\kappa^{2} c(z)^{2}}} .
$$

- The random complex coefficient $\mathcal{T}_{\omega, \kappa}^{\varepsilon}$ is the generalized transmission coefficient for the pressure release boundary conditions. It can be expressed in terms of the standard mode-dependent reflection and transmission coefficients of the random medium (i.e. with matched or transparent boundary conditions) $R_{\omega, \kappa}^{\varepsilon}$ and $T_{\omega, \kappa}^{\varepsilon}$, that are introduced in appendix A:

$$
\mathcal{T}_{\omega, \kappa}^{\varepsilon}=\exp \left(-i \frac{\omega}{\varepsilon} \tau(\kappa,-L)\right) \frac{T_{\omega, \kappa}^{\varepsilon}}{1-R_{\omega, \kappa}^{\varepsilon}},
$$

for $\tau(\kappa, z)$ the vertical mode-dependent travel time

$$
\tau(\kappa, z)=\int_{0}^{z} \frac{d z^{\prime}}{c\left(\kappa, z^{\prime}\right)} .
$$

We describe the statistial properties of the generalized transmission coefficient $\mathcal{T}_{\omega, \kappa}^{\varepsilon}$ in the next subsection. We remark here that the expansion

$$
\mathcal{T}_{\omega, \kappa}^{\varepsilon}=\sum_{j=0}^{\infty} \exp \left(-i \frac{\omega}{\varepsilon} \tau(\kappa,-L)\right) T_{\omega, \kappa}^{\varepsilon}\left(R_{\omega, \kappa}^{\varepsilon}\right)^{j},
$$

reflects the physical situation that the waves recorded at the surface are the sum of the contributions of the waves that have been directly transmitted from the source plane $z=-L$ to the surface $z=0$ and the contributions of the waves that have reached the surface and have then been reflected back and forth by the surface and by the random region generating "multiples". These reflections and multiples arise due to the pressure release boundary condition $(j=0)$ at the surface which is an important ingredient in our model.

- The integral in $\kappa$ is limited to $\kappa \leq \kappa_{\max }$ where $\kappa_{\max }$ is defined by

$$
\kappa_{\max }=\min _{z \in(-L, 0)} c^{-1}(z) .
$$


The condition $\kappa<\kappa_{\max }$ means that there is no turning point for the wave mode with slowness $\kappa$. Modes generated with transverse slownesses that violate this condition do not reach the surface, so they can be neglected (remember that the random medium is layered so that scattering does not change the slowness).

4.2. Statistical Analysis of the Reflection and Transmission Coefficients. The integrated covariance $\gamma(z)$ of the fluctuations of the random medium is defined by

$$
\gamma(z)=\int_{-\infty}^{\infty} \mathbb{E}\left[\nu(z, 0) \nu\left(z, z^{\prime}\right)\right] d z^{\prime}
$$

As we will see below it plays the role of a depth-dependent scattering coefficient. The following proposition describes the statistical properties of the generalized transmission coefficient and it is proved in [11]. It is an extension of the well-known results in the case of matched boundary conditions [8, Chapter 14-15].

Proposition 4.1. The autocorrelation function of the generalized transmission coefficient at two nearby slownesses satisfies

$$
\mathbb{E}\left[\mathcal{T}_{\omega, \kappa+\varepsilon \lambda / 2}^{\varepsilon} \overline{\mathcal{T}_{\omega, \kappa-\varepsilon \lambda / 2}^{\varepsilon}}\right] \stackrel{\varepsilon \rightarrow 0}{\longrightarrow} \int \mathcal{U}(\omega, \kappa, \xi) \exp (-i \omega \kappa \lambda \xi) d \xi
$$

The spectral density $\mathcal{U}(\omega, \kappa, \xi)$ has the probabilistic representation:

$$
\begin{aligned}
\mathcal{U}(\omega, \kappa, \xi)= & \mathbb{E}\left[\delta\left(\xi-\int_{-L}^{0} c(\kappa, z)\left(2 N_{\omega, \kappa}(z)+1\right) d z\right)\right. \\
& \left.\times \exp \left(-\int_{-L}^{0} \frac{\sigma(z) c(\kappa, z)}{\zeta(z) c(z)}\left(2 N_{\omega, \kappa}(z)+1\right) d z\right) \mid N_{\omega, \kappa}(-L)=0\right]
\end{aligned}
$$

where $\left(N_{\omega, \kappa}(z)\right)_{-L \leq z \leq 0}$ is a jump Markov process with state space $\mathbb{N}$ and inhomogeneous infinitesimal generator

$$
\mathcal{L}_{z} \phi(N)=\frac{\gamma(z) c(\kappa, z)^{2} \omega^{2}}{4 c(z)^{4}}\left[(N+1)^{2}(\phi(N+1)-\phi(N))+N^{2}(\phi(N-1)-\phi(N))\right]
$$

If the medium has no random fluctuations $\nu \equiv 0$, then the Markov process does not jump and we find that

$$
\mathcal{U}(\omega, \kappa, \xi)=\delta\left(\xi-\int_{-L}^{0} c(\kappa, z) d z\right) \exp \left(-\int_{-L}^{0} \frac{\sigma(z) c(\kappa, z)}{\zeta(z) c(z)} d z\right)
$$

which allows us to recover the results of Section 3 .

If the medium is not dissipative $\sigma \equiv 0$, then

$$
\mathcal{U}(\omega, \kappa, \xi)=\mathbb{E}\left[\delta\left(\xi-\int_{-L}^{0} c(\kappa, z)\left(2 N_{\omega, \kappa}(z)+1\right) d z\right) \mid N_{\omega, \kappa}(-L)=0\right],
$$

which shows in particular that:

$$
\lim _{\varepsilon \rightarrow 0} \mathbb{E}\left[\left|\mathcal{T}_{\omega, \kappa}^{\varepsilon}\right|^{2}\right]=\int_{0}^{\infty} \mathcal{U}(\omega, \kappa, \xi) d \xi=1
$$

This energy conservation relation is easy to understand in the case of a homogeneous medium, in which the wave is simply transmitted so that indeed $\left|\mathcal{T}_{\omega, \kappa}^{\varepsilon}\right|=1$. In the 
general random case the relation (4.14) is a deep result that is analyzed in detail in [11]. It is a reflection of the fact that the pressure release boundary conditions and the randomly layered medium traps the wave in a surface layer giving a waveguide effect.

In general, for a fixed pair $(\omega, \kappa)$, the function $\xi \rightarrow \mathcal{U}(\omega, \kappa, \xi)$ is a distribution with a singular (Dirac) component corresponding to the directly transmitted waves and an absolutely continuous component corresponding to wave energy that has undergone multiple scattering.

LEMMA 4.2. We have

$$
\mathcal{U}(\omega, \kappa, \xi)=\mathcal{U}_{s}(\omega, \kappa, \xi)+\mathcal{U}_{c}(\omega, \kappa, \xi),
$$

with

$$
\begin{array}{r}
\mathcal{U}_{s}(\omega, \kappa, \xi)=\delta\left(\xi-\int_{-L}^{0} c(\kappa, z) d z\right) \exp \left(-\int_{-L}^{0} \frac{\sigma(z) c(\kappa, z)}{\zeta(z) c(z)} d z\right) \\
\times \exp \left(-\int_{-L}^{0} \frac{\gamma(z) c^{2}(\kappa, z) \omega^{2}}{4 c(z)^{4}} d z\right)
\end{array}
$$

and

$$
\begin{aligned}
\mathcal{U}_{c}(\omega, \kappa, \xi)= & \exp \left(-\int_{-L}^{0} \frac{\sigma(z) c(\kappa, z)}{\zeta(z) c(z)} d z\right) \\
\times & \int_{-L}^{0} d z_{0} \frac{\gamma\left(z_{0}\right) c^{2}\left(\kappa, z_{0}\right) \omega^{2}}{4 c^{4}\left(z_{0}\right)} \exp \left(-\int_{-L}^{z_{0}} \frac{\gamma(z) c^{2}(\kappa, z) \omega^{2}}{4 c^{4}(z)} d z\right) \\
& \times \mathbb{E}\left[\delta\left(\xi-2 \int_{z_{0}}^{0} c(\kappa, z) N_{\omega, \kappa}(z) d z-\int_{-L}^{0} c(\kappa, z) d z\right)\right. \\
& \left.\times \exp \left(-2 \int_{z_{0}}^{0} \frac{\sigma(z) c(\kappa, z)}{\zeta(z) c(z)} N_{\omega, \kappa}(z) d z\right) \mid N_{\omega, \kappa}\left(z_{0}\right)=1\right] .
\end{aligned}
$$

The singular component in (4.15) can be obtained from the probabilistic representation (4.12) as the contributions of the trajectories that do not jump. In the wave problem this is the contributions of the waves that would constitute the wave front if the sources were emitting short pulses.

The continuous component in (4.16) can also be obtained from the probabilistic representation (4.12), this is the contributions of the trajectories that jump at least once. In the wave problem this is the contributions of the waves that would constitute the coda wave if the sources were emitting short pulses. Since $N_{\omega, \kappa}$ takes nonnegative values, the support of the continuous component $\mathcal{U}_{c}$ in $\xi$ is $\left[\int_{-L}^{0} c(\kappa, z) d z, \infty\right)$. In the case of a weakly scattering medium, i.e.

$$
\int_{-L}^{0} \frac{\gamma(z) c^{2}(\kappa, z) \omega^{2}}{4 c^{4}(z)} d z \ll 1,
$$

we have

$$
\begin{aligned}
& \mathcal{U}_{c}(\omega, \kappa, \xi) \simeq \exp \left(-\int_{-L}^{0} \frac{\sigma(z) c(\kappa, z)}{\zeta(z) c(z)} d z\right) \int_{-L}^{0} \frac{\gamma\left(z_{0}\right) c^{2}\left(\kappa, z_{0}\right) \omega^{2}}{4 c^{4}\left(z_{0}\right)} \\
& \quad \times \delta\left(\xi-2 \int_{z_{0}}^{0} c(\kappa, z) d z-\int_{-L}^{0} c(\kappa, z) d z\right) \exp \left(-2 \int_{z_{0}}^{0} \frac{\sigma(z) c(\kappa, z)}{\zeta(z) c(z)} d z\right) d z_{0}
\end{aligned}
$$


In the probabilistic interpretation this is the contribution of paths that jump exactly once. In the wave problem this corresponds to the first order reflections from the medium, that is, of the directly transmitted wave that is reflected at the surface with the pressure release boundary condition and finally returned to the surface again by scattering induced by the microscale fluctuations. $\mathrm{s}$

5. The Cross Correlation in a Random Medium. The next proposition is the fundamental result that gives the relation between the statistical cross correlation and the spectral density $\mathcal{U}$.

Proposition 5.1. The statistical cross correlation is given by

$$
\begin{array}{r}
C^{\varepsilon}\left(-\varepsilon s, \boldsymbol{x}+\frac{\varepsilon \boldsymbol{y}}{2}, \boldsymbol{x}-\frac{\varepsilon \boldsymbol{y}}{2}\right)=\frac{1}{(2 \pi)^{5}} \iiint D^{\varepsilon}(\omega, \boldsymbol{\kappa}, \boldsymbol{\lambda}) \hat{F}(\omega) \hat{G}(\omega \boldsymbol{\kappa}) \hat{\theta}(\omega \boldsymbol{\lambda}) \\
\quad \times \mathcal{T}_{\omega,|\boldsymbol{\kappa}+\varepsilon \boldsymbol{\lambda} / 2|}^{\varepsilon} \frac{\mathcal{T}_{\omega,|\boldsymbol{\kappa}-\varepsilon \boldsymbol{\lambda} / 2|}^{\varepsilon}}{\exp (-i \omega(s-\boldsymbol{\kappa} \cdot \boldsymbol{y}-\boldsymbol{\lambda} \cdot \boldsymbol{x})) \omega^{4} d \boldsymbol{\kappa} d \boldsymbol{\lambda} d \omega},
\end{array}
$$

where

$$
\begin{aligned}
& D^{\varepsilon}(\omega, \boldsymbol{\kappa}, \boldsymbol{\lambda})=\frac{\alpha_{x} \omega^{2}\left(\kappa^{2}-\varepsilon^{2} \lambda^{2} / 4\right)}{\omega^{2} \rho(-L)^{2}+\varepsilon^{2} \sigma(-L)^{2}} \frac{\zeta^{1 / 2}(|\boldsymbol{\kappa}+\varepsilon \boldsymbol{\lambda} / 2|,-L) \zeta^{1 / 2}(|\boldsymbol{\kappa}-\varepsilon \boldsymbol{\lambda} / 2|,-L)}{\zeta^{1 / 2}(|\boldsymbol{\kappa}+\varepsilon \boldsymbol{\lambda} / 2|, 0) \zeta^{1 / 2}(|\boldsymbol{\kappa}-\varepsilon \boldsymbol{\lambda} / 2|, 0)} \\
& +\frac{\alpha_{z}}{\zeta^{1 / 2}(|\boldsymbol{\kappa}+\varepsilon \boldsymbol{\lambda} / 2|, 0) \zeta^{1 / 2}(|\boldsymbol{\kappa}-\varepsilon \boldsymbol{\lambda} / 2|, 0) \zeta^{1 / 2}(|\boldsymbol{\kappa}+\varepsilon \boldsymbol{\lambda} / 2|,-L) \zeta^{1 / 2}(|\boldsymbol{\kappa}-\varepsilon \boldsymbol{\lambda} / 2|,-L)} .
\end{aligned}
$$

In the regime $\varepsilon \rightarrow 0$, the cross correlation is self-averaging with respect to the distribution of the random medium and

$$
C^{\varepsilon}\left(-\varepsilon s, \boldsymbol{x}+\frac{\varepsilon \boldsymbol{y}}{2}, \boldsymbol{x}-\frac{\varepsilon \boldsymbol{y}}{2}\right) \stackrel{\varepsilon \rightarrow 0}{\longrightarrow} \mathcal{C}(s, \boldsymbol{x}, \boldsymbol{y}),
$$

in probability, where

$$
\begin{aligned}
\mathcal{C}(s, \boldsymbol{x}, \boldsymbol{y})= & \frac{1}{(2 \pi)^{3}} \iint D(\kappa) \hat{F}(\omega) \hat{G}(\omega \boldsymbol{\kappa})\left[\int \theta(\boldsymbol{x}-\xi \boldsymbol{\kappa}) \mathcal{U}(\omega, \kappa, \xi) d \xi\right] \\
& \times \exp (-i \omega(s-\boldsymbol{\kappa} \cdot \boldsymbol{y})) \omega^{2} d \boldsymbol{\kappa} d \omega \\
D(\kappa)= & \frac{\zeta(\kappa,-L)}{\zeta(\kappa, 0) \rho(-L)^{2}}\left[\frac{\alpha_{z}}{c(-L)^{2}}+\left(\alpha_{x}-\alpha_{z}\right) \kappa^{2}\right] .
\end{aligned}
$$

Proof. We first substitute the integral representation (4.5) into the expression of $C^{\varepsilon}$ :

$$
\begin{aligned}
C^{\varepsilon}\left(t, \boldsymbol{x}_{1}, \boldsymbol{x}_{2}\right)=\frac{1}{(2 \pi)^{5} \varepsilon^{2}} \iiint \hat{F}(\omega) \hat{G}\left(\omega \frac{\boldsymbol{\kappa}_{1}+\boldsymbol{\kappa}_{2}}{2}\right) \hat{\theta}\left(\frac{\omega\left(\boldsymbol{\kappa}_{1}-\boldsymbol{\kappa}_{2}\right)}{\varepsilon}\right) e^{i \frac{\omega}{\varepsilon}\left(t+\boldsymbol{\kappa}_{1} \cdot \boldsymbol{x}_{1}-\boldsymbol{\kappa}_{2} \cdot \boldsymbol{x}_{2}\right)} \\
\quad \times \mathcal{T}_{\omega, \kappa_{1}}^{\varepsilon} \frac{\mathcal{T}_{\omega, \kappa_{2}}^{\varepsilon}}{\mathcal{T}^{\varepsilon}}\left[\frac{\alpha_{x} \omega^{2} \boldsymbol{\kappa}_{1} \cdot \boldsymbol{\kappa}_{2}}{\omega^{2} \rho(-L)^{2}+\varepsilon^{2} \sigma(-L)^{2}} \frac{\zeta^{1 / 2}\left(\kappa_{1},-L\right) \zeta^{1 / 2}\left(\kappa_{2},-L\right)}{\zeta^{1 / 2}\left(\kappa_{1}, 0\right) \zeta^{1 / 2}\left(\kappa_{2}, 0\right)}\right. \\
\left.+\frac{\alpha_{z}}{\zeta^{1 / 2}\left(\kappa_{1},-L\right) \zeta^{1 / 2}\left(\kappa_{2},-L\right) \zeta^{1 / 2}\left(\kappa_{1}, 0\right) \zeta^{1 / 2}\left(\kappa_{2}, 0\right)}\right] \omega^{4} d \boldsymbol{\kappa}_{1} d \boldsymbol{\kappa}_{2} d \omega .
\end{aligned}
$$

Next we change of variables $\boldsymbol{\kappa}_{1}=\boldsymbol{\kappa}+\varepsilon \boldsymbol{\lambda} / 2, \boldsymbol{\kappa}_{2}=\boldsymbol{\kappa}-\varepsilon \boldsymbol{\lambda} / 2$ :

$$
\begin{aligned}
C^{\varepsilon}\left(t, \boldsymbol{x}_{1}, \boldsymbol{x}_{2}\right)=\frac{1}{(2 \pi)^{5}} & \iiint \hat{F}(\omega) \hat{G}(\omega \boldsymbol{\kappa}) \hat{\theta}(\omega \boldsymbol{\lambda}) D^{\varepsilon}(\omega, \boldsymbol{\kappa}, \boldsymbol{\lambda}) \mathcal{T}_{\omega,|\boldsymbol{\kappa}+\varepsilon \boldsymbol{\lambda} / 2|}^{\varepsilon} \frac{\boldsymbol{T}_{\omega,|\boldsymbol{\kappa}-\varepsilon \boldsymbol{\lambda} / 2|}^{\varepsilon}}{\varepsilon} \\
& \times \exp \left(i \omega \frac{t}{\varepsilon}+i \omega \boldsymbol{\kappa} \cdot \frac{\boldsymbol{x}_{1}-\boldsymbol{x}_{2}}{\varepsilon}+i \omega \boldsymbol{\lambda} \cdot \frac{\boldsymbol{x}_{1}+\boldsymbol{x}_{2}}{2}\right) \omega^{4} d \boldsymbol{\kappa} d \boldsymbol{\lambda} d \omega .
\end{aligned}
$$


If we take $t=-\varepsilon s, \quad \boldsymbol{x}_{1}=\boldsymbol{x}+\varepsilon \boldsymbol{y} / 2$, and $\boldsymbol{x}_{2}=\boldsymbol{x}-\varepsilon \boldsymbol{y} / 2$, then we obtain (5.1). The decoherence frequency of the reflection coefficient is of order $\varepsilon$ [8, Chapter 9], and the decoherence (transverse) wavenumber is also of order $\varepsilon$ [8, Chapter 14], so the integral over a frequency band of order one and a wavenumber band of order one is self-averaging (with respect to the distribution of the random medium) in the regime $\varepsilon \rightarrow 0$ we then find:

$$
\begin{aligned}
C^{\varepsilon}\left(-\varepsilon s, \boldsymbol{x}+\frac{\varepsilon \boldsymbol{y}}{2}\right. & \left., \boldsymbol{x}-\frac{\varepsilon \boldsymbol{y}}{2}\right) \stackrel{\varepsilon \rightarrow 0}{\longrightarrow} \frac{1}{(2 \pi)^{5}} \iiint \hat{F}(\omega) \lim _{\varepsilon \rightarrow 0} \mathbb{E}\left[\mathcal{T}_{\omega,|\boldsymbol{\kappa}+\varepsilon \boldsymbol{\lambda} / 2|}^{\varepsilon} \overline{\left.\mathcal{T}_{\omega,|\boldsymbol{\kappa}-\varepsilon \boldsymbol{\lambda} / 2|}^{\varepsilon}\right]}\right. \\
& \times D(\kappa) \hat{G}(\omega \boldsymbol{\kappa}) \hat{\theta}(\omega \boldsymbol{\lambda}) \exp (-i \omega(s-\boldsymbol{\kappa} \cdot \boldsymbol{y}-\boldsymbol{\lambda} \cdot \boldsymbol{x})) \omega^{4} d \boldsymbol{\kappa} d \boldsymbol{\lambda} d \omega .
\end{aligned}
$$

Using Proposition 4.1 we obtain (5.3).

That the cross correlations are statistically stable and not "noisy" is important when using them as data for imaging. We next make some comments regarding the phenomenon of statistical stability.

- The proposition shows that the cross correlation is statistically stable with respect to the distribution of the sources and with respect to the distribution of the random medium, provided that $T$ is large and $\varepsilon$ is small.

- On the one hand, $T$ is a parameter that can be chosen by the user. Statistical stability with respect to the distribution of the sources can be obtained by integrating over a long time window, which can always be done provided the signals can be recorded over arbitrarily long times and that we can assume that the noise sources are stationary.

- On the other hand, $\varepsilon$ is a parameter that depends on the medium and on the bandwidth of the sources. It cannot be chosen by the user. If $\varepsilon$ is not small, then statistical stability with respect to the distribution of the random medium is not achieved and the cross correlation function will exhibit fluctuations around the asymptotic value (5.3). As a result one should average over different realizations of the medium and/or average over the space and time lags $\boldsymbol{y}$ and $s$.

6. Inverse Problem. In this section we show that it is possible to estimate the cross spectral density $\mathcal{U}$ from the observation of the cross correlation $C^{\varepsilon}$ in the regime $\varepsilon \rightarrow 0$. Note that the cross correlation $C^{\varepsilon}$ is non-zero only for small offsets corresponding to $t$ and $\boldsymbol{x}-\boldsymbol{x}^{\prime}$ being of order $\varepsilon$. The inverse problem is therefore very different from surface tomography problems that has been considered in many cases involving wave field correlations so far. In these tomography problems the cross correlations give travel time estimates which subsequently are used for tomographic estimation of velocity maps. In the present case we have to make the link in between the macroscopic aspects of the medium and the local correlations in order to solve the inverse problem. This link is indeed given by the cross spectral density $\mathcal{U}$ which contains all information about the medium that we can derive from the correlations.

We first present a relation between a regularized Fourier transform of the cross correlation $C^{\varepsilon}$ and the spectral density $\mathcal{U}$. If we take a Fourier transform in $\boldsymbol{y}, \boldsymbol{x}$, and $s$ :

$$
\widehat{C}^{\varepsilon}(\omega, \boldsymbol{\kappa}, \boldsymbol{\lambda}):=\iiint C^{\varepsilon}\left(-\varepsilon s, \boldsymbol{x}+\frac{\varepsilon \boldsymbol{y}}{2}, \boldsymbol{x}-\frac{\varepsilon \boldsymbol{y}}{2}\right) \exp (i \omega(s-\boldsymbol{\kappa} \cdot \boldsymbol{y}-\boldsymbol{\lambda} \cdot \boldsymbol{x})) d s d \boldsymbol{y} d \boldsymbol{x},
$$

then we obtain for any $\varepsilon$ :

$$
\widehat{C}^{\varepsilon}(\omega, \boldsymbol{\kappa}, \boldsymbol{\lambda})=\hat{F}(\omega) \hat{G}(\omega \boldsymbol{\kappa}) \hat{\theta}(\omega \boldsymbol{\lambda}) \mathcal{T}_{\omega,|\boldsymbol{\kappa}+\varepsilon \boldsymbol{\lambda} / 2|} \overline{\mathcal{T}_{\omega,|\boldsymbol{\kappa}-\varepsilon \boldsymbol{\lambda} / 2|}^{\varepsilon}} D^{\varepsilon}(\omega, \boldsymbol{\kappa}, \boldsymbol{\lambda}) .
$$


This quantity is not self-averaging (with respect to the distribution of the random medium) in the regime $\varepsilon \rightarrow 0$, but its expectation converges as $\varepsilon \rightarrow 0$ :

$$
\mathbb{E}\left[\widehat{C}^{\varepsilon}(\omega, \boldsymbol{\kappa}, \boldsymbol{\lambda})\right] \stackrel{\varepsilon \rightarrow 0}{\longrightarrow} \hat{F}(\omega) \hat{G}(\omega \boldsymbol{\kappa}) \hat{\theta}(\omega \boldsymbol{\lambda}) D(\kappa)\left[\int \exp (-i \omega \boldsymbol{\kappa} \cdot \boldsymbol{\lambda} \xi) \mathcal{U}(\omega, \kappa, \xi) d \xi\right],
$$

and its decoherence frequency and wavenumber are of order $\varepsilon$. Therefore, if we convolve (or smooth) $\widehat{C}^{\varepsilon}(\omega, \boldsymbol{\kappa}, \boldsymbol{\lambda})$ in $\omega$ and $\boldsymbol{\kappa}$ with a kernel whose radius is large compared to $\varepsilon$ but small compared to 1 , for instance

$$
\widehat{C}_{s}^{\varepsilon}(\omega, \boldsymbol{\kappa}, \boldsymbol{\lambda}):=\frac{1}{\pi^{3 / 2} \varepsilon^{3 p}} \iint \widehat{C}^{\varepsilon}(\tilde{\omega}, \tilde{\boldsymbol{\kappa}}, \boldsymbol{\lambda}) \exp \left(-\frac{|\omega \boldsymbol{\kappa}-\tilde{\omega} \tilde{\boldsymbol{\kappa}}|^{2}}{\varepsilon^{2 p}}-\frac{|\omega-\tilde{\omega}|^{2}}{\varepsilon^{2 p}}\right) \tilde{\omega}^{2} d \tilde{\boldsymbol{\kappa}} d \tilde{\omega}
$$

with $p \in(0,1)$, then we obtain in the regime $\varepsilon \rightarrow 0$ :

$$
\lim _{\varepsilon \rightarrow 0} \widehat{C}_{s}^{\varepsilon}(\omega, \boldsymbol{\kappa}, \boldsymbol{\lambda})=\hat{F}(\omega) \hat{G}(\omega \boldsymbol{\kappa}) \hat{\theta}(\omega \boldsymbol{\lambda}) D(\kappa)\left[\int \exp (-i \omega \boldsymbol{\kappa} \cdot \boldsymbol{\lambda} \xi) \mathcal{U}(\omega, \kappa, \xi) d \xi\right]
$$

in probability. This gives the expression of the smoothed Fourier transform $\widehat{C}_{s}^{\varepsilon}$ of the cross correlation in terms of the spectral density $\mathcal{U}$ in the regime $\varepsilon \rightarrow 0$. We next show how we can get a more explicit expression for the spectral density.

First, we will carry out a deconvolution step to remove the effect of the random sources:

Definition 6.1. Let us define

$$
\widehat{V}^{\varepsilon}(\omega, \boldsymbol{\kappa}, \boldsymbol{\lambda}):=\frac{\widehat{C}_{s}^{\varepsilon}(\omega, \boldsymbol{\kappa}, \boldsymbol{\lambda})}{\widehat{C}_{s}^{\varepsilon}(\omega, \boldsymbol{\kappa}, \mathbf{0})} \frac{\widehat{C}_{s}^{\varepsilon}(\omega, \mathbf{0}, \mathbf{0})}{\widehat{C}_{s}^{\varepsilon}(\omega, \mathbf{0}, \boldsymbol{\lambda})} .
$$

The deconvolved data is not available for all values of the parameters and to characterize the available observations we define $\Omega$ by:

DeFinition 6.2. Let us define

$$
\Omega=\left\{(\omega, \kappa) \in \mathbb{R} \times \mathbb{R}^{+} \mid \hat{F}(\omega)>0 \text { and } \hat{G}(\omega \tilde{\boldsymbol{\kappa}})>0 \text { for a.e. } \tilde{\boldsymbol{\kappa}} \text { s.t. }|\tilde{\boldsymbol{\kappa}}|=\kappa\right\} \text {. }
$$

We next preset the important result that shows how we can relate the observations to the spectral kernel for the available observations when we make an additional assumption of locality of $\theta$ :

Proposition 6.3. Assume that the vertical velocity field is observed everywhere at the surface, and that $\theta$ satisfies:

$$
\hat{\theta}(\boldsymbol{k}) \neq 0 \text { for almost every } \boldsymbol{k} \text {. }
$$

1. Define $\mathcal{V}(\omega, \kappa, \xi)$ by

$$
\mathcal{V}(\omega, \kappa, \xi)=\frac{\mathcal{U}(\omega, \kappa, \xi)}{\int \mathcal{U}\left(\omega, \kappa, \xi^{\prime}\right) d \xi^{\prime}}
$$

for $(\omega, \kappa) \in \Omega$.

2. The density

$$
V^{\varepsilon}(\omega, \kappa, \xi):=\frac{1}{(2 \pi)^{3}} \int_{\partial B(\mathbf{0}, \kappa)} \int_{\partial B(\mathbf{0}, \kappa \xi)} \int \exp (i \omega \tilde{\boldsymbol{\lambda}} \cdot \boldsymbol{x}) \widehat{V}^{\varepsilon}(\omega, \tilde{\boldsymbol{\kappa}}, \tilde{\boldsymbol{\lambda}}) \omega^{2} d \tilde{\boldsymbol{\lambda}} d \sigma(\boldsymbol{x}) d \sigma(\tilde{\boldsymbol{\kappa}}),
$$


is such that

$$
\lim _{\varepsilon \rightarrow 0} V^{\varepsilon}(\omega, \kappa, \xi)=\mathcal{V}(\omega, \kappa, \xi)
$$

Proof. Since $\hat{\theta}$ is non-zero almost everywhere, the quantity $\widehat{V}^{\varepsilon}(\omega, \boldsymbol{\kappa}, \boldsymbol{\lambda})$ defined by (6.6) is well defined for $(\omega, \boldsymbol{\kappa})$ in the support of $\hat{F}(\omega) \hat{G}(\omega \boldsymbol{\kappa})$, (the expression (6.5) shows that the denominator is positive for $\varepsilon$ small enough). Its limit as $\varepsilon \rightarrow 0$ is given by

$$
\lim _{\varepsilon \rightarrow 0} \widehat{V}^{\varepsilon}(\omega, \boldsymbol{\kappa}, \boldsymbol{\lambda})=\frac{\int \exp (-i \omega \boldsymbol{\kappa} \cdot \boldsymbol{\lambda} \xi) \mathcal{U}(\omega, \kappa, \xi) d \xi}{\int \mathcal{U}(\omega, \kappa, \xi) d \xi}=\int \exp (-i \omega \boldsymbol{\kappa} \cdot \boldsymbol{\lambda} \xi) \mathcal{V}(\omega, \kappa, \xi) d \xi
$$

Taking the special inverse Fourier transform (6.6), and using the identity

$$
\int_{\partial B(\mathbf{0}, \kappa)} \int_{\partial B(\mathbf{0}, \kappa \xi)} \delta(\boldsymbol{x}-\tilde{\boldsymbol{\kappa}} \tilde{\xi}) d \sigma(\boldsymbol{x}) d \sigma(\tilde{\boldsymbol{\kappa}})=2 \pi \delta(\xi-\tilde{\xi})
$$

we obtain the desired result (6.11).

We remark here that:

- We can only estimate $\mathcal{U}(\omega, \kappa, \xi)$ for $(\omega, \kappa) \in \Omega$, that is, the pairs $(\omega, \kappa)$ (frequency, transverse wavenumber) in the support of the waves emitted by the noise sources). Therefore, the bandwidth of the noise sources must be large enough so that the procedure to extract the background velocity and scattering coefficient can be applied to the estimate of $\mathcal{V}(\omega, \kappa, \xi)$ when restricted to $(\omega, \kappa) \in \Omega$.

- The condition (6.8) is important. If, for instance, $\theta \equiv 1$, that is, if the noise sources are distributed uniformly, then we get from (5.3) that

$\mathcal{C}(s, \boldsymbol{x}, \boldsymbol{y})=\frac{1}{(2 \pi)^{3}} \iint D(\kappa) \hat{F}(\omega) \hat{G}(\omega \boldsymbol{\kappa}) \exp (-i \omega(s-\boldsymbol{\kappa} \cdot \boldsymbol{y}))\left[\int \mathcal{U}(\omega, \kappa, \xi) d \xi\right] \omega^{2} d \boldsymbol{\kappa} d \omega$,

which shows that we have access to the integrated cross spectral density only. This is not enough to reconstruct the parameters of the medium. Indeed, we have seen that in a non-dissipative medium the integrated cross spectral density (4.14) is equal to one, whatever the background velocity or scattering properties.

- If the medium is not dissipative, then the integrated cross spectral density (4.14) is equal to one and therefore $\mathcal{V}$ is equal to $\mathcal{U}$, which means that indeed $\mathcal{V}^{\varepsilon}$ is an estimator of $\mathcal{U}$.

\section{Numerical Examples.}

7.1. Dissipative Statistically Homogeneous Random Medium. Before we discuss an important model estimation problem in Section 7.2, we describe here the the situation when the medium is statistically homogeneous and dissipative in the random medium section $(-L, 0)$. This allows us to illustrate the performance of and principle behind the spectral estimation. Thus, we assume the model: $c(z)=c_{0}$, $\sigma(z)=\sigma_{0}$, and $\gamma(z)=\gamma_{0}$. In this case the asymptotic spectral density is given by (4.12) and we have in the asymptotic regime $\varepsilon \rightarrow 0$ :

$$
\begin{aligned}
& \mathbb{E}\left[\mathcal{T}_{\omega, \kappa+\varepsilon \lambda / 2}^{\varepsilon} \overline{\mathcal{T}_{\omega, \kappa-\varepsilon \lambda / 2}^{\varepsilon}}\right] \stackrel{\varepsilon \rightarrow 0}{\longrightarrow} \exp \left(-\left(i \omega \kappa \lambda+\frac{\sigma_{0}}{\zeta_{0} c_{0}}\right) c_{0}(\kappa) L\right) \\
& \times \mathbb{E}\left[\exp \left(-2\left(i \omega \kappa \lambda+\frac{\sigma_{0}}{\zeta_{0} c_{0}}\right) c_{0}(\kappa) \int_{-L}^{0} N_{\omega, \kappa}(z) d z\right)\right],
\end{aligned}
$$


where $\left(N_{\omega, \kappa}(z)\right)_{-L \leq z \leq 0}$ is the jump Markov process starting from $N_{\omega, \kappa}(-L)=0$ with the homogeneous infinitesimal generator:

$$
\mathcal{L}_{\omega, \kappa} \phi(N)=\frac{\gamma_{0} c_{0}^{2}(\kappa) \omega^{2}}{4 c_{0}^{4}}\left[(N+1)^{2}(\phi(N+1)-\phi(N))+N^{2}(\phi(N-1)-\phi(N))\right] .
$$

In the numerical simulations we use a stepwise constant layered medium. The thickness of the elementary layers is $l_{c}=0.05$. The density of the medium is one, the bulk modulus takes values $1 /\left(1 \pm \delta_{\kappa}\right)$ independently in each layer with probability $1 / 2$, with $\delta_{\kappa}=0.5$. The absorption coefficient is $\sigma_{0}=0.005$. The thickness of the random medium is $L=500$. The dissipative distance is $L_{d i s}=\zeta_{0} /\left(2 \sigma_{0}\right)=$ 100. The localization length for the frequency $\omega=1$ and slowness $\kappa=0$ is $L_{l o c}=$ $\left(4 c_{0}^{2}\right) /\left(\delta_{\kappa}^{2} l_{c} \omega^{2}\right)^{-1}=320$. We refer to [8] for a detailed discussion of the localization length and its significance. It is sufficient here to observe that it articulates the penetration depth of the wave energy. The sources are a collection of point sources emitting independent stationary random signals with temporal power spectral density $\hat{F}(\omega)$ supported in $|\omega| \in(0.5,2)$. The typical wavelength $\lambda_{0}$ is therefore of the order of 5 and the ratio $l_{c} / \lambda_{0} \simeq \lambda_{0} / L \simeq 0.01$. These values are of the same order as the ones encountered in exploration seismology [17], in which the decoherence length is $2 \mathrm{~m}$, the typical wavelength is $150 \mathrm{~m}$, and the probing depth of the order of $10 \mathrm{~km}$.

We perform a series of numerical simulations with different realizations of the random medium. The vertical velocity field is observed at the surface. We compute the empirical cross correlation function $C_{T}^{\varepsilon}$ and its Fourier transform $\widehat{C}_{T}^{\varepsilon}$ :

$$
\widehat{C}_{T}^{\varepsilon}(\omega, \boldsymbol{\kappa}, \boldsymbol{\lambda}):=\iiint C_{T}^{\varepsilon}\left(-s, \boldsymbol{x}+\frac{\boldsymbol{y}}{2}, \boldsymbol{x}-\frac{\boldsymbol{y}}{2}\right) \exp (i \omega(s-\boldsymbol{\kappa} \cdot \boldsymbol{y}-\boldsymbol{\lambda} \cdot \boldsymbol{x})) d s d \boldsymbol{y} d \boldsymbol{x},
$$

for $|\boldsymbol{\lambda}| \leq \lambda_{\max }=0.09,|\boldsymbol{\kappa}| \leq \kappa_{\max }=0.9$, and $|\omega| \leq \omega_{\max }=2$. For $T$ large enough $\widehat{C}_{T}^{\varepsilon}(\omega, \boldsymbol{\kappa}, \boldsymbol{\lambda})$ is an estimator for

$$
\widehat{C}^{\varepsilon}(\omega, \boldsymbol{\kappa}, \boldsymbol{\lambda})=\frac{\alpha}{\zeta_{0}^{2}} \hat{F}(\omega) \hat{G}(\omega \boldsymbol{\kappa}) \hat{\theta}(\omega \boldsymbol{\lambda}) \mathcal{T}_{\omega,|\boldsymbol{\kappa}+\boldsymbol{\lambda} / 2|}^{\varepsilon} \overline{\mathcal{T}_{\omega,|\boldsymbol{\kappa}-\boldsymbol{\lambda} / 2|}^{\varepsilon}} .
$$

Recall that this quantity is not statistically stable with respect to the medium fluctuations. Therefore, we smooth in $\omega$ (with a "frequency bandwidth" of 0.15 ) and in $\kappa$ (with a "slowness bandwidth" of 0.1 ) as in (6.4) so that we obtain $\widehat{C}_{T, s}^{\varepsilon}$ and we then look at

$$
\widehat{V}_{T}^{\varepsilon}(\omega, \boldsymbol{\kappa}, \boldsymbol{\lambda})=\frac{\widehat{C}_{T, s}^{\varepsilon}(\omega, \boldsymbol{\kappa}, \boldsymbol{\lambda})}{\widehat{C}_{T, s}^{\varepsilon}(\omega, \mathbf{0}, \boldsymbol{\lambda})} \frac{\widehat{C}_{T, s}^{\varepsilon}(\omega, \mathbf{0}, \mathbf{0})}{\widehat{C}_{T, s}^{\varepsilon}(\omega, \boldsymbol{\kappa}, \mathbf{0})}
$$

which is an estimator (for large $T$ and small $\varepsilon$ ) of

$$
\begin{aligned}
& \widehat{\mathcal{V}}(\omega, \boldsymbol{\kappa}, \boldsymbol{\lambda})=\frac{\int \exp (-i \omega \boldsymbol{\kappa} \cdot \boldsymbol{\lambda} \xi) \mathcal{U}(\omega, \kappa, \xi) d \xi}{\int \mathcal{U}(\omega, \kappa, \xi) d \xi} \\
& =\frac{\mathbb{E}\left[\exp \left(-2\left(i \omega \boldsymbol{\kappa} \cdot \boldsymbol{\lambda}+\frac{\sigma_{0}}{\zeta_{0} c_{0}}\right) c_{0}(\kappa) \int_{-L}^{0} N_{\omega, \kappa}(z) d z\right)\right]}{\mathbb{E}\left[\exp \left(-2 \frac{\sigma_{0}}{\zeta_{0} c_{0}} c_{0}(\kappa) \int_{-L}^{0} N_{\omega, \kappa}(z) d z\right)\right]} \exp \left(-i \omega \boldsymbol{\kappa} \cdot \boldsymbol{\lambda} c_{0}(\kappa) L\right)
\end{aligned}
$$

The approximate identity $\widehat{V}_{T}^{\varepsilon}(\omega, \boldsymbol{\kappa}, \boldsymbol{\lambda}) \simeq \widehat{\mathcal{V}}(\omega, \boldsymbol{\kappa}, \boldsymbol{\lambda})$ would be an equality in the asymptotic regime $\varepsilon \rightarrow 0, T \rightarrow \infty$, while in particular $\varepsilon$ is finite in our numerical set up 
a)
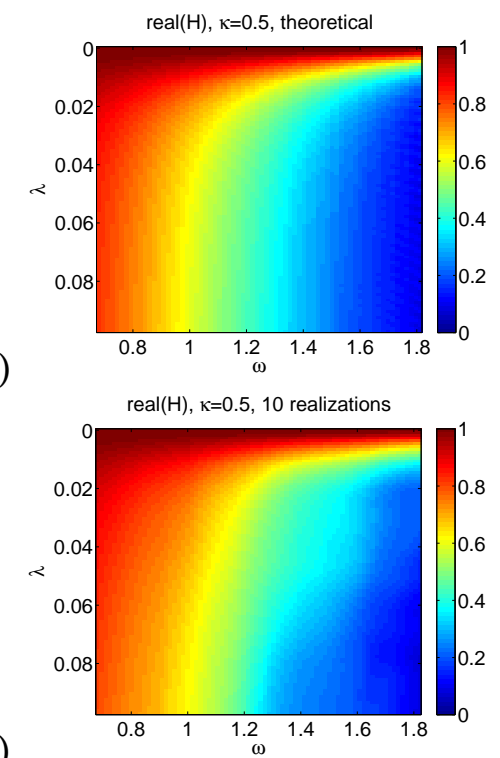

b)
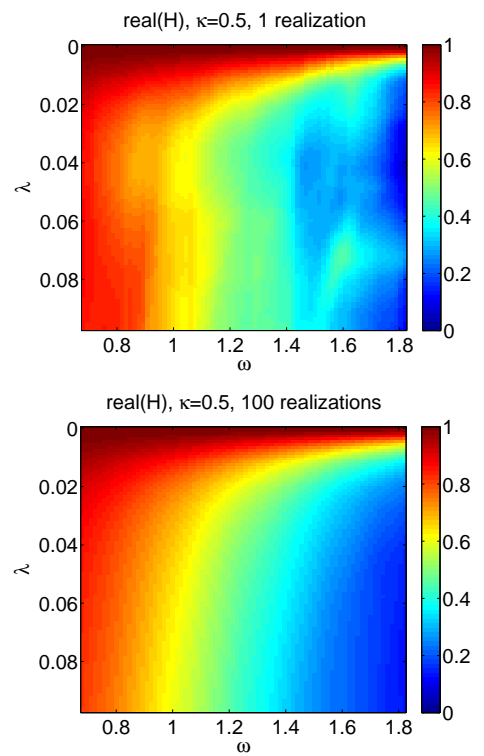

FIG. 7.1. Real parts of the theoretical function $\widehat{\mathcal{H}}(\omega, \boldsymbol{\kappa}, \boldsymbol{\lambda})$ (a) and of the empirical function $\widehat{H}_{T}^{\varepsilon}(\omega, \boldsymbol{\kappa}, \boldsymbol{\lambda})(b-c-d)$. The empirical function is plotted without averaging over the random medium (b), with an averaging over 10 realizations of the random medium (c), and with an averaging over 100 realizations of the random medium (d).

as in any real situation. The separation of scales in our configuration is not large enough to give statistical stability of the estimation of the spectral density. As we now show there is still statistical fluctuations in the estimated quantities after the local smoothing in $\boldsymbol{\kappa}$ and $\omega$.

In the homogeneous case we have

$$
\widehat{\mathcal{V}}(\omega, \boldsymbol{\kappa}, \boldsymbol{\lambda})=\exp \left(-i \omega \boldsymbol{\kappa} \cdot \boldsymbol{\lambda} c_{0}(\kappa) L\right) .
$$

We will therefore plot in the figures the normalized functions

$$
\begin{aligned}
\widehat{H}_{T}^{\varepsilon}(\omega, \boldsymbol{\kappa}, \boldsymbol{\lambda}) & =\exp \left(i \omega \boldsymbol{\kappa} \cdot \boldsymbol{\lambda} c_{0}(\kappa) L\right) \widehat{V}_{T}^{\varepsilon}(\omega, \boldsymbol{\kappa}, \boldsymbol{\lambda}), \\
\widehat{\mathcal{H}}(\omega, \boldsymbol{\kappa}, \boldsymbol{\lambda}) & =\exp \left(i \omega \boldsymbol{\kappa} \cdot \boldsymbol{\lambda} c_{0}(\kappa) L\right) \widehat{\mathcal{V}}(\omega, \boldsymbol{\kappa}, \boldsymbol{\lambda}) .
\end{aligned}
$$

In Figure 7.1-7.2a we plot the (real and imaginary) parts of the theoretical function $\widehat{\mathcal{H}}(\omega, \boldsymbol{\kappa}, \boldsymbol{\lambda})$ defined by $(7.6)$ for $\boldsymbol{\kappa}=(0.5,0)$ and $\boldsymbol{\lambda}=\lambda(1,0)$ as a function of $\omega$ and $\lambda$. In Figure 7.1-7.2b we plot the (real and imaginary) parts of the estimator $\widehat{H}_{T}^{\varepsilon}(\omega, \boldsymbol{\kappa}, \boldsymbol{\lambda})$ for one realization of the random medium. In Figure 7.1-7.2c (respectively $\mathrm{d}$ ) we plot the (real and imaginary) parts of the estimator $\widehat{H}_{T}^{\varepsilon}(\omega, \boldsymbol{\kappa}, \boldsymbol{\lambda})$ averaged over 10 (respectively 100) realizations of the random medium. We can see that the agreement is qualitative without averaging, quantitatively good after an averaging over 10 realizations and excellent after an averaging over 100 realizations.

The agreement between theoretical formulas and empirical ones becomes better when one looks at quantities integrated over $\omega$ and/or $\kappa$. For instance, even the empirical function $\left\langle\widehat{H}_{T}^{\varepsilon}(\cdot, \boldsymbol{\kappa}, \boldsymbol{\lambda})\right\rangle_{\omega}$ integrated over $\omega$ without averaging with respect to realizations is close to the theoretical function $\langle\widehat{\mathcal{H}}(\cdot, \boldsymbol{\kappa}, \boldsymbol{\lambda})\rangle_{\omega}$ (see Figure 7.3). We will further illustrate this fact in the next section. 
a)
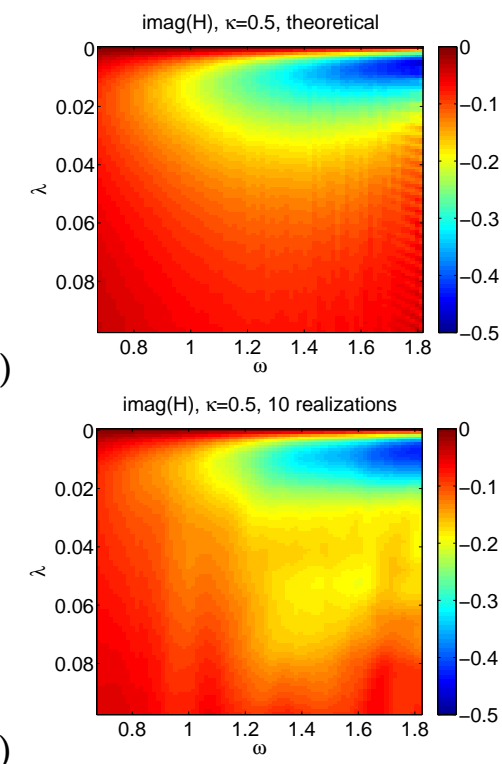

b)
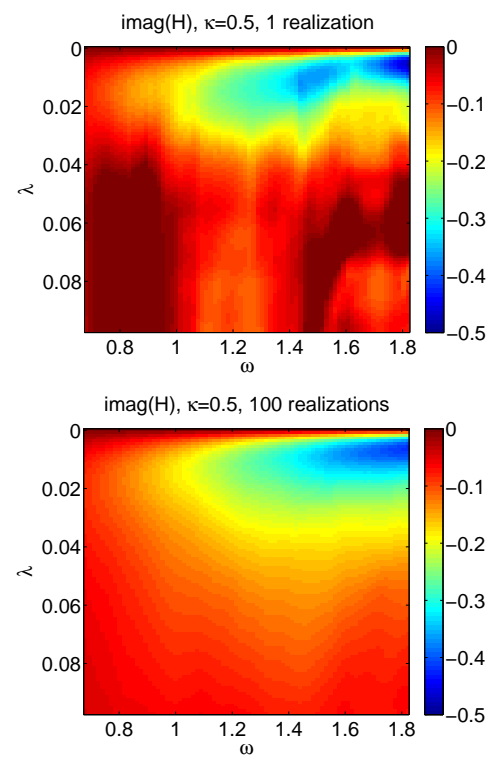

FIG. 7.2. Same as in Fig. 7.1 but for the imaginary parts of the theoretical function $\widehat{\mathcal{H}}(\omega, \boldsymbol{\kappa}, \boldsymbol{\lambda})$ (a) and of the empirical function $\widehat{H}_{T}^{\varepsilon}(\omega, \boldsymbol{\kappa}, \boldsymbol{\lambda})(b-c-d)$. The empirical function is plotted without averaging over the random medium (b), with an averaging over 10 realizations of the random medium (c), and with an averaging over 100 realizations of the random medium (d).

a)

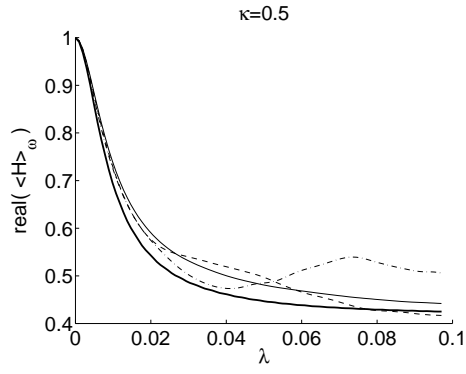

b)

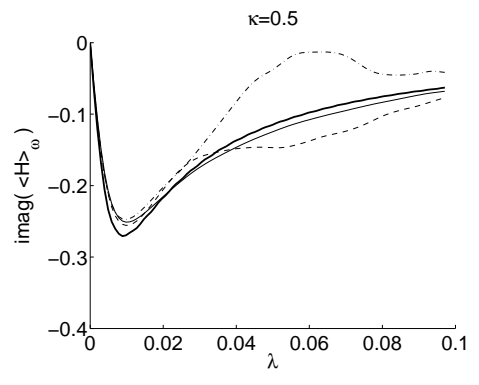

FIG. 7.3. Real (a) and imaginary (b) parts of the theoretical function $\langle\widehat{\mathcal{H}}(\cdot, \boldsymbol{\kappa}, \boldsymbol{\lambda})\rangle_{\omega}$ (thick solid lines) and of the empirical function $\left\langle\widehat{H}_{T}^{\varepsilon}(\cdot, \boldsymbol{\kappa}, \boldsymbol{\lambda})\right\rangle$ integrated over $\omega$ without averaging (dot-dashed lines) with averaging over 10 realizations (dashed lines), and with averaging over 100 realizations (thin solid lines).

As an application of the results in this section, if we deal with a statistically homogeneous and dissipative medium it is possible to extract the scattering and absorption parameters from a least-square fit of the theoretical integrated function to the empirical integrated function.

7.2. Detection of an Interface. We describe here the detection of a weak interface, an interface without coherent reflections. The medium is assumed to have piecewise constant macroscale medium parameters. We assume that the background velocity and impedance are constant in the whole section $(-L, 0)$ and that the random medium $(-L, 0)$ consists of two different regions $\left(-L,-z_{s}\right)$ and $\left(-z_{s}, 0\right)$. The 
dissipation and integrated covariance jump at the interface

$$
\sigma(z)=\left\{\begin{array}{l}
\sigma_{0} \text { if } z \in\left(-z_{s}, 0\right) \\
\sigma_{1} \text { if } z \in\left(-L,-z_{s}\right)
\end{array} \quad \gamma(z)=\left\{\begin{array}{l}
\gamma_{0} \text { if } z \in\left(-z_{s}, 0\right) \\
0 \text { if } z \in\left(-L,-z_{s}\right)
\end{array}\right.\right.
$$

Here $\sigma_{0}$ and $\sigma_{1}$ may be equal and we assume $\gamma_{0}>0$. Thus, we can in particular consider the situation when the interface only distinguishes itself via a jump in the scattering coefficient, that is, a change in the fluctuation magnitude and or spatial decoherence length of the microscale medium fluctuations from a situation with vanishingly small fluctuations. Note that we assume that the top region is scattering which conforms with typical situations in the earth's crust with relative stronger scattering in the surface layer. As already mentioned we suppose that there is no impedance and background velocity contrast $(\zeta(z)$ and $c(z)$ are constant and equal to $\zeta_{0}$ and $\left.c_{0}\right)$. We remark that a jump in these quantities typically would make the inverse problem simpler since one could then exploit traveltime dependent features in the recordings for imaging purposes. In our situation we want to use cross correlations of the recorded field to estimate the position $z_{s}$ of the interface. We now describe the theoretical asymptotic behavior of the spectral density in the situation described.

LEMMA 7.1. If the top layer is weakly scattering $\gamma_{0} \omega^{2} z_{s} /\left(4 c_{0}\right) \ll 1$, then we have

$$
\mathcal{U}(\omega, \kappa, \xi)=\mathcal{U}_{s}(\omega, \kappa, \xi)+\mathcal{U}_{c}(\omega, \kappa, \xi)
$$

with the singular and continuous components given by

$$
\begin{aligned}
& \mathcal{U}_{s}(\omega, \kappa, \xi)=\exp \left(-\frac{c_{0}(\kappa)\left[\sigma_{0} z_{s}+\sigma_{1}\left(L-z_{s}\right)\right]}{\zeta_{0} c_{0}}-\frac{\gamma_{0} c_{0}(\kappa)^{2} \omega^{2} z_{s}}{4 c_{0}^{4}}\right) \delta\left(\xi-c_{0}(\kappa) L\right), \\
& \mathcal{U}_{c}(\omega, \kappa, \xi) \simeq \frac{\gamma_{0} \omega^{2}}{8 c_{0}^{2}} \exp \left(-\frac{\sigma_{0} \xi+c_{0}(\kappa)\left(\sigma_{1}-\sigma_{0}\right)\left(L-z_{s}\right)}{\zeta_{0} c_{0}}\right) \mathbf{1}_{\left[c_{0}(\kappa) L, c_{0}(\kappa)\left(L+2 z_{s}\right)\right]}(\xi)
\end{aligned}
$$

The normalized density (6.9) is also of the form

$$
\mathcal{V}(\omega, \kappa, \xi)=\mathcal{V}_{s}(\omega, \kappa, \xi)+\mathcal{V}_{c}(\omega, \kappa, \xi)
$$

where the singular and continuous components are given by

$$
\begin{aligned}
& \mathcal{V}_{s}(\omega, \kappa, \xi) \simeq \delta\left(\xi-c_{0}(\kappa) L\right) \\
& \mathcal{V}_{c}(\omega, \kappa, \xi) \simeq \frac{\gamma_{0} \omega^{2}}{8 c_{0}^{2}} \exp \left(-\frac{\sigma_{0}\left(\xi-c_{0}(\kappa) L\right)}{\zeta_{0} c_{0}}\right) \mathbf{1}_{\left[0,2 c_{0}(\kappa) z_{s}\right]}\left(\xi-c_{0}(\kappa) L\right)
\end{aligned}
$$

This shows that

i) the support of the singular component (corresponding to the direct waves) of the cross spectral density gives the depth of the sources,

ii) the support of the continuous component (corresponding to the multiply-scattered waves) of the cross spectral density gives the depth of the interface.

From the theoretical point of view, it seems straightforward to get the depth of the interface. However, from the practical point of view, statistical stability (with respect to the distribution of the random medium) is an issue and it is necessary to integrate over $\omega$ and $\kappa$. As a consequence it is not easy to detect the jumps and the support of the cross spectral density. It is in fact easier to work with the modified functions $\widehat{H}_{T}^{\varepsilon}$ and $\widehat{\mathcal{H}}$ as we now explain. 
If the top region in $\left(-z_{s}, 0\right)$ in weakly scattering and weakly dissipative, in the sense that $\gamma_{0} \omega^{2} z_{s} /\left(4 c_{0}\right) \ll 1$ and $\sigma_{0} z_{s} / \zeta_{0} \ll 1$, then we have for $\widehat{\mathcal{H}}$ defined as in $(7.6)$ :

$\widehat{\mathcal{H}}(\omega, \boldsymbol{\kappa}, \boldsymbol{\lambda}) \simeq 1-\frac{\gamma_{0} \omega^{2} c_{0}^{2}(\kappa) z_{s}}{4 c_{0}^{4}}+\frac{\gamma_{0} \omega^{2} c_{0}^{2}(\kappa) z_{s}}{4 c_{0}^{4}} \frac{\sin \left(\omega \boldsymbol{\kappa} \cdot \boldsymbol{\lambda} c_{0}(\kappa) z_{s}\right)}{\omega \boldsymbol{\kappa} \cdot \boldsymbol{\lambda} c_{0}(\kappa) z_{s}} \exp \left(-i \omega \boldsymbol{\kappa} \cdot \boldsymbol{\lambda} c_{0}(\kappa) z_{s}\right)$.

Note in particular that $\omega^{-2} c_{0}^{-2}(\kappa) \operatorname{Im}(\widehat{\mathcal{H}}(\omega, \boldsymbol{\kappa}, \boldsymbol{\lambda}))$ depends on $(\omega, \boldsymbol{\kappa}, \boldsymbol{\lambda})$ only through the product $\omega \boldsymbol{\kappa} \cdot \boldsymbol{\lambda} c_{0}(\kappa)$. This remark indicates how we should integrate over $(\omega, \boldsymbol{\kappa}, \boldsymbol{\lambda})$ in order to get a statistically stable quantity that can give a robust estimate of the depth of the interface as described in step 3) below.

If we assume that the vertical velocity field is recorded everywhere at the surface, then we can get the empirical cross correlation $C_{T}^{\varepsilon}$. The practical identification of the interface is as follows:

1) we compute from the recorded signals the smoothed Fourier transform of the cross correlation $\widehat{C}_{T, s}^{\varepsilon}$ defined by (7.1) with a smoothing procedure as described just below (7.1). We then compute the empirical function $\widehat{V}_{T, s}^{\varepsilon}$ defined by (7.2).

2) we extract $\widehat{H}_{T}^{\varepsilon}(\omega, \boldsymbol{\kappa}, \boldsymbol{\lambda})$ as in $(7.5)$, which is an estimator of $\widehat{\mathcal{H}}(\omega, \boldsymbol{\kappa}, \boldsymbol{\lambda})$.

3) we compute

$$
W_{T}^{\varepsilon}(k)=-\frac{\iiint \omega^{-2} c_{0}^{-2}(\kappa) \operatorname{Im}\left(\widehat{H}_{T}^{\varepsilon}(\omega, \kappa, \boldsymbol{\lambda})\right) \delta\left(k-\omega c_{0}(\kappa) \boldsymbol{\kappa} \cdot \boldsymbol{\lambda}\right) d \boldsymbol{\kappa} d \boldsymbol{\lambda} d \omega}{\iiint \delta\left(k-\omega c_{0}(\kappa) \boldsymbol{\kappa} \cdot \boldsymbol{\lambda}\right) d \boldsymbol{\kappa} d \boldsymbol{\lambda} d \omega} .
$$

Here the integration is carried out over frequencies $\omega$ and transverse wavenumbers $\kappa$ for which we can estimate the spectral density, i.e. $(\omega, \kappa) \in \Omega$ where $\Omega$ is defined by (6.7). By a comparison (for instance, a least-square fit) with the theoretical formula:

$$
\begin{aligned}
\mathcal{W}(k) & =-\frac{\iiint \omega^{-2} c_{0}^{-2}(\kappa) \operatorname{Im}(\widehat{\mathcal{H}}(\omega, \kappa, \boldsymbol{\lambda})) \delta\left(k-\omega c_{0}(\kappa) \boldsymbol{\kappa} \cdot \boldsymbol{\lambda}\right) d \boldsymbol{\kappa} d \boldsymbol{\lambda} d \omega}{\iiint \delta\left(k-\omega c_{0}(\kappa) \boldsymbol{\kappa} \cdot \boldsymbol{\lambda}\right) d \boldsymbol{\kappa} d \boldsymbol{\lambda} d \omega} \\
& =\frac{\gamma_{0} z_{s}}{4 c_{0}^{4}} \frac{\sin ^{2}\left(k z_{s}\right)}{k z_{s}}
\end{aligned}
$$

we can estimate the position of the interface $z_{s}$. We now apply this idea.

The numerical setup is the following. The randomly layered medium is stepwise constant. The thickness of the elementary layers is $l_{c}=0.05$. The density is one, the bulk modulus takes values $1 /\left(1 \pm \delta_{\kappa}\right)$ independently in each layer with probability $1 / 2$, the parameter $\delta_{\kappa}=0.1$ for $z \in\left(-z_{s}, 0\right)$ and $\delta_{\kappa}=0$ for $z \in\left(-L,-z_{s}\right)$, with $L=500$ and $z_{s}=100$. Therefore $\gamma_{0}=\delta_{\kappa}^{2} l_{c}=0.0005$. The background velocity and impedance are both equal to one, and the dissipation coefficient is constant $\sigma_{0}=\sigma_{1}=0.0005$. This means that the interface is characterized by a jump in the statistical properties of the medium fluctuations only. Note that the dissipation distance $L_{d i s}=\zeta_{0} /\left(2 \sigma_{0}\right)=$ 1000 and the localization length $\left(4 c_{0}^{2}\right) /\left(\gamma_{0} \omega^{2}\right)=2000$ for $\omega=2$ are both larger than $z_{s}$, which means that the approximate formula (7.8) for the theoretical function $\widehat{\mathcal{H}}$ is relevant.

We have performed 1000 simulations. For each of them we compute the function $W_{T}^{\varepsilon}(k)$. Note that we do not average over the realizations of the random medium. In Figure $7.4 \mathrm{a}$ we plot the theoretical function $\mathcal{W}(k)$ and the empirical functions $W_{T}^{\varepsilon}(k)$ corresponding to the first 100 simulations. One can see that the empirical functions are reasonably stable, in particular the location of the minimum is always close to the theoretical value. Motivated by this observation, we propose to use this minimum 
a)

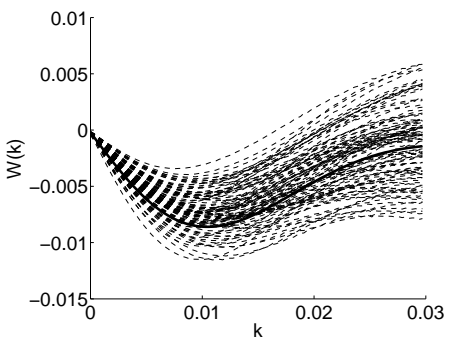

b)

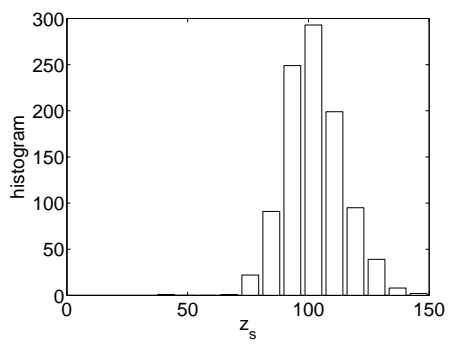

FIG. 7.4. Picture a: Empirical functions $k \mapsto W_{T}^{\varepsilon}(k)$ (dashed lines) and theoretical function $k \rightarrow \mathcal{W}(k)$ (thick solid line). Picture b: Histogram of the estimated values of the depth of the interface over 1000 simulations. The real depth is $z_{s}=100$.

to estimate the depth of the interface. In Figure $7.4 \mathrm{~b}$ we plot the histogram of the estimations, whose numerical mean value is 102 and numerical standard deviation is 11. This means that the proposed method for the estimation of the depth of the interface has an accuracy of the order of $10 \%$.

8. Summary. We summarize here the main aspects of the framework that we have set forth.

Deep-Earth Noise Sources. The sources in our problem are a zero-mean stationary in time random field located at the depth $z=-L$. The spectrum of the noise sources is given in (2.3). In our context $F$ plays the role of source wavelet, $G$ describes the spatial source coherence, $\theta$ gives the spatial locality of the noise sources and $\boldsymbol{\alpha}$ corresponds to a source directivity vector. Here we make an assumption about separability, that is, factorization into microscale coherence factors $(F$ and $G)$ and a macroscale modulation $(\theta)$ factor. This property is used in the source deconvolution step described below. We also assume spatial diversity of the noise source probing, so that in particular $\theta$ is not constant, only then will the data contain useful information about the medium.

Scale Separation. From (2.3) it is clear that the decoherence time and length of the random sources are of order $\varepsilon \ll 1$. This corresponds to a situation with high-frequency waves. We also assume that the microscale random fluctuations have a characteristic scale of variation which is $\varepsilon^{2}$, while the depth of the random section $L$ is $\mathcal{O}(1)$. This separation of scales assumption enables us to make use of asymptotic results to describe the wave field correlations via diffusion or white-noise approximations [8] combined with a high-frequency analysis and from this we can get expressions for the wave field spectrum, as in Proposition 4.1.

Measurements and Second Moment Information. A main aspect of our framework is that we use incoherent waves generated by deep random noise sources and emerging at the surface from a randomly layered medium to image for the macroscale parameters of the medium. The observations are therefore incoherent and we convert them to the empirical cross correlations in (2.4) in order to extract useful medium information. We assume that the recording time $T$ is large so that the empirical cross correlations can be replaced by the statistical cross correlations in (2.6). We remark that in our idealized setup we assume observations everywhere on the surface. The case with more restricted sampling would entail a degradation of resolution and stability. 
Quantities of Interest. The quantities of interest are the macroscopic medium properties, that is, the background or effective medium local speed of sound $c(z)$, the effective impedance $\zeta(z)$, the dissipation $\sigma(z)$, and the scattering coefficient $\gamma(z)$ of the random microstructure, see Section 4 . We assume that the background impedance $\zeta(z)$ is smooth. This means that there are no strong coherent reflectors in our formulation, the main scattering is the one associated with the microstructure. Here, the background velocity is also chosen to be smooth, see [8, Chapter 11] for an example with an inverse problem in the context where this has a jump.

Source Deconvolution and Mesoscale Smoothing. A main result in the paper is the expression (5.3) for the correlation data (in the limit of separation of scales). The spectral kernel $\mathcal{U}$ gives the second moment of the generalized transmission coefficient $\mathcal{T}^{\varepsilon}$ (in the limit of separation of scales) that in turn gives the coupling of the medium to the vertical velocity at the surface. This kernel contains all useful information about the medium that can be obtained from the correlation data. To elucidate this information we carry out the specific Fourier transform in (6.1). We have now identified the medium information more explicitly, but at a cost. The resulting information, given in (6.2), directly gives the cross moment of the generalized transmission coefficient, however, it is noisy and exhibits statistical fluctuations around its mean in (6.3). To reduce the fluctuations around the mean we therefore carry out an a priori mesoscale smoothing step as in (6.4), exploiting rapid decoherence in frequency and wavenumber. In order to articulate the medium information further we then compute the source deconvolution in (6.6), to remove the effect of the source. To "lay bare" the spectral kernel itself we next carry out the specific Fourier inverse in (6.10). We have then arrived at the important preprocessed imaging data. The theoretical expression for the imaging data is given in (6.9). We see that indeed this is essentially the spectral kernel that we aimed to identify, it is exactly so in the non-dissipative case. The further processing depends on the specific imaging objective and our prior hypothesis for the structure of the macroscale that we want to identify, we discuss the specific case of interface detection in the paper. We stress however that until this point the framework is general and can be used in the context of other imaging problems. Note that in [1] it is shown how knowledge of a spectral kernel can be used for estimation of smooth background parameters. We remark however here that the principal challenge in practical imaging is that the imaging data is not statistically stable due to a finite scale separation, this essentially means that we are not quite in the averaging limit of the imaging data to its mean in (6.9).

Background Deconvolution and Interface Imaging. The underlying theoretical result of this paper is described in Section 4.2. It describes the autocorrelation function of the generalized transmission coefficient in terms of the spectral density $\mathcal{U}$. This can be used to relate the imaging functional to macroscopic medium features and guide the construction of the imaging functional. At present inverting for a general background profile seems challenging due to lack of statistical stability of imaging data for realistic levels of scale separation. It is however remarkable that we can invert for the location of a jump in the scattering coefficient assuming a constant background profile $\left(c_{0}, \zeta_{0}\right)$. This procedure takes the quantity $\widehat{V}_{T}^{\varepsilon}$ defined by $(7.2)$ as it starting point. We deconvolve with respect to the background, that is, we divide by its value in the case without microscale fluctuations, given in (7.4), to form the source and background deconvolved data $\widehat{H}_{T}^{\varepsilon}$. The main challenge is to regain statistical stability and, rather than inverting for the spectral kernel, we are able to integrate partially in $\boldsymbol{\kappa}, \boldsymbol{\lambda}$ and $\omega$ in a way that is intended to create optimal stability while 
leaving information about the location of the jump intact to create $W_{T}^{\varepsilon}$ in (7.9). We finally remark that the imaginary part of $\widehat{H}_{T}^{\varepsilon}$ is most sensitive to the location and the one we use in creating $W_{T}^{\varepsilon}$, in fact this corresponds to filtering out the directly transmitted wave and retaining the imaginary part of the regular spectral component that exhibits the interface location.

We remark that the framework we have presented can be generalized to the case with random sources at the surface. The coinage backlight has been used for this configuration [10]. The situation can be analyzed in terms of analytic expressions for the spectral density of the associated reflection operators [8], with the corresponding kernel replacing $\mathcal{U}$, however, the image processing parallels the one presented here. The situation with weak interface zones and imaging of these in the case of ambient noise can be handled using an adaption of the techniques presented in [11]. In the paper [11] is also presented a further discussion of the spectral density $\mathcal{U}$, which indeed is the central quantity in the context of imaging with incoherent waves.

9. Conclusion. In this paper we have investigated how information about the background medium and macroscale parameters can be retrieved from cross correlations of noisy signals when the medium is randomly layered. We have considered the situation when the noise sources are located in the subsurface and the wave fields are recorded at the surface. The recorded wave fields are the waves transmitted through the medium and they contain information about the slowly varying background and the statistical properties of the small-scale random fluctuations.

We have shown that it is theoretically possible to reconstruct the slowly varying background velocity, scattering coefficient and dissipation parameter if the bandwidth of the noise sources is large enough and if the spatial support function $\theta(\boldsymbol{x})$ of the noise sources satisfies the condition $\hat{\theta}(\boldsymbol{k}) \neq 0$ for almost every $\boldsymbol{k}$. This result holds in an asymptotic framework where the decoherence length of the medium is much smaller than the typical wavelength of the signals generated by the noise sources, which is itself much smaller than the macroscopic scale of variation of the background medium. If, for instance, the spatial support of the noise sources is uniform, then it is not possible to reconstruct the background medium.

We have emphasized in our discussion that an important practical issue is statistical stability. The cross correlation is statistically stable with respect to the distribution of the sources, provided the integration time is large enough. However the statistical stability of the cross correlation with respect to the distribution of the random medium requires a large separation of scales between the decoherence length of the random fluctuations of the medium and the typical wavelength of the noise sources on the one hand, and a large separation of scales between the typical wavelength of the noise sources and the macroscopic scale of variations of the background medium on the other hand. This large separation of scales may not be achieved in practice and it is therefore desirable to seek ways of averaging to regain statistical stability. In typical situations averaging over the medium is not possible. There is only one realization of the random earth say. We remark that there may be situations in which the medium is not perfectly layered and in which the microscale variations decorrelate faster than the background changes, so that one can exploit averaging over measurements with lateral offsets. This situation is however delicate, the full analysis of such a situation is an open question. What is important here is that we have been able to show that even in the case with a perfectly layered model and scale parameters that are motivated by typical acquisition situations we have been able to stabilize the data and invert for medium parameters. This we have achieved via averaging over 
frequency and wavenumbers. In conclusion, we have proposed a robust algorithms to detect an interface in the medium and we have shown that it is possible to retrieve the interface position under reasonable conditions. Detection of such interfaces or boundaries in between geological facies is an important model problem in seismology. From the theoretical viewpoint much more is however possible. We have shown that in fact the whole (assumed smooth) background velocity profile of the medium can be reconstructed from the observations of the incoherent waves measured at the surface that is induced by the (statistically stationary in time) noise sources that are located deep inside the medium. What prevents us from exploiting this theoretical result at present is the lack of statistical stability with respect to the medium fluctuations in situations where the scale separations are chosen to reflect typical acquisition configurations. The reconstruction of an arbitrary background velocity profile is thus possible in theory, albeit seems difficult in practice, unless several independent realizations of the medium can be exploited for averaging.

Appendix A. Derivation of the Integral Representation of the Recorded Field. We use the same approach as in [8] using a propagator formulation. We first take a Fourier transform in the time and transverse spatial coordinates:

$$
\begin{aligned}
& \hat{p}^{\varepsilon}(\omega, \boldsymbol{\kappa}, z)=\iint p^{\varepsilon}(t, \boldsymbol{x}, z) e^{i \frac{\omega}{\varepsilon}(t-\boldsymbol{\kappa} \cdot \boldsymbol{x})} d t d \boldsymbol{x}, \\
& \hat{u}^{\varepsilon}(\omega, \boldsymbol{\kappa}, z)=\iint u^{\varepsilon}(t, \boldsymbol{x}, z) e^{i \frac{\omega}{\varepsilon}(t-\boldsymbol{\kappa} \cdot \boldsymbol{x})} d t d \boldsymbol{x} .
\end{aligned}
$$

We next introduce the right- and left-going modes defined by

$$
\begin{aligned}
& \hat{a}^{\varepsilon}(\omega, \kappa, z)=\left(\frac{1}{\sqrt{\zeta(\kappa, z)}} \hat{p}^{\varepsilon}(\omega, \kappa, z)+\sqrt{\zeta(\kappa, z)} \hat{u}^{\varepsilon}(\omega, \kappa, z)\right) e^{-i \frac{\omega}{\varepsilon} \tau(\kappa, z)} \\
& \hat{b}^{\varepsilon}(\omega, \kappa, z)=\left(-\frac{1}{\sqrt{\zeta(\kappa, z)}} \hat{p}^{\varepsilon}(\omega, \kappa, z)+\sqrt{\zeta(\kappa, z)} \hat{u}^{\varepsilon}(\omega, \kappa, z)\right) e^{i \frac{\omega}{\varepsilon} \tau(\kappa, z)} .
\end{aligned}
$$

The pressure release boundary condition is $\hat{p}^{\varepsilon}=0$, or equivalently

$$
\hat{a}^{\varepsilon}(\omega, \kappa, 0)=\hat{b}^{\varepsilon}(\omega, \kappa, 0) .
$$

The jump conditions across the source plane at $z=-L$ are

$$
\left[\hat{p}^{\varepsilon}\right]_{(-L)^{-}}^{(-L)^{+}}=\hat{f}_{z}, \quad\left[\hat{u}^{\varepsilon}\right]_{(-L)^{-}}^{(-L)^{+}}=\frac{i \omega}{\rho(-L) i \omega-\varepsilon \sigma(-L)} \kappa \cdot \hat{\boldsymbol{f}}_{\boldsymbol{x}}
$$

which gives with the radiation condition $\hat{a}^{\varepsilon}\left(\omega, \kappa,(-L)^{-}\right)=0$ :

$$
\hat{a}^{\varepsilon}\left(\omega, \boldsymbol{\kappa},(-L)^{+}\right)=\left(\frac{\hat{f}_{z}}{\sqrt{\zeta(\kappa,-L)}}+\frac{i \omega \sqrt{\zeta(\kappa,-L)}}{\rho(-L) i \omega-\varepsilon \sigma(-L)} \kappa \cdot \boldsymbol{f}_{\boldsymbol{x}}\right)(\omega, \boldsymbol{\kappa}) e^{-i \frac{\omega}{\varepsilon} \tau(\kappa,-L)}
$$

We introduce the propagator matrix, that is the $2 \times 2$ matrix such that, for any $-L \leq z_{0} \leq z \leq 0$

$$
\mathbf{P}^{\varepsilon}\left(\omega, \boldsymbol{\kappa}, z_{0}, z\right)\left[\begin{array}{l}
\hat{a}^{\varepsilon}\left(\omega, \boldsymbol{\kappa}, z_{0}\right) \\
\hat{b}^{\varepsilon}\left(\omega, \boldsymbol{\kappa}, z_{0}\right)
\end{array}\right]=\left[\begin{array}{l}
\hat{a}^{\varepsilon}(\omega, \boldsymbol{\kappa}, z) \\
\hat{b}^{\varepsilon}(\omega, \boldsymbol{\kappa}, z)
\end{array}\right]
$$

The propagator matrix is the solution of the linear system

$$
\frac{d \mathbf{P}^{\varepsilon}}{d z}=\mathbf{M}^{\varepsilon}(\omega, \kappa, z) \mathbf{P}^{\varepsilon}
$$




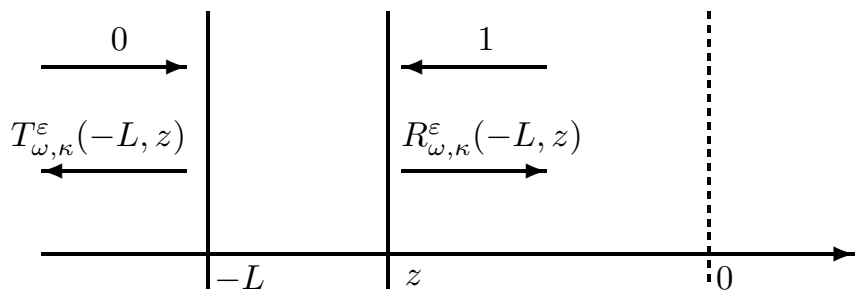

FIG. A.1. Reflection and transmission coefficients.

with the initial condition $\mathbf{P}^{\varepsilon}\left(\omega, \boldsymbol{\kappa}, z_{0}, z_{0}\right)=\mathbf{I}$ and the $2 \times 2$ matrix $\mathbf{M}^{\varepsilon}$ given by

$$
\begin{aligned}
& M_{11}^{\varepsilon}(z)=\frac{i \omega c(\kappa, z)}{2 c^{2}(z) \varepsilon} \nu\left(z, \frac{z}{\varepsilon^{2}}\right)-\frac{\sigma(z)}{2 \zeta(z)} \frac{c(\kappa, z)}{c(z)}, \\
& M_{21}^{\varepsilon}(z)=\left[\frac{i \omega c(\kappa, z)}{2 c^{2}(z) \varepsilon} \nu\left(z, \frac{z}{\varepsilon^{2}}\right)-\frac{\sigma(z)}{2 \zeta(z)} \frac{c(\kappa, z)}{c(z)}\left(1-2 c^{2}(z) \kappa^{2}\right)+\frac{\zeta^{\prime}(\kappa, z)}{2 \zeta(\kappa, z)}\right] e^{2 i \frac{\omega}{\varepsilon} \tau(\kappa, z)}, \\
& M_{12}(z)=\left[-\frac{i \omega c(\kappa, z)}{2 c^{2}(z) \varepsilon} \nu\left(z, \frac{z}{\varepsilon^{2}}\right)+\frac{\sigma(z)}{2 \zeta(z)} \frac{c(\kappa, z)}{c(z)}\left(1-2 c^{2}(z) \kappa^{2}\right)+\frac{\zeta^{\prime}(\kappa, z)}{2 \zeta(\kappa, z)}\right] e^{-2 i \frac{\omega}{\varepsilon} \tau(\kappa, z),} \\
& M_{22}^{\varepsilon}(z)=-\frac{i \omega c(\kappa, z)}{2 c^{2}(z) \varepsilon} \nu\left(z, \frac{z}{\varepsilon^{2}}\right)+\frac{\sigma(z)}{2 \zeta(z)} \frac{c(\kappa, z)}{c(z)}=-M_{11}^{\varepsilon}(z) .
\end{aligned}
$$

Note that $\mathbf{P}^{\varepsilon}\left(\omega, \boldsymbol{\kappa}, z_{0}, z\right)$ depends on $\boldsymbol{\kappa}$ only through the modulus $\kappa=|\boldsymbol{\kappa}|$.

We introduce the transmission and reflection coefficients $\left(T_{\omega, \kappa}^{\varepsilon}, R_{\omega, \kappa}^{\varepsilon}\right)$ defined by the relation

$$
\mathbf{P}^{\varepsilon}(\omega, \kappa,-L, z)\left[\begin{array}{c}
0 \\
T_{\omega, \kappa}^{\varepsilon}(-L, z)
\end{array}\right]=\left[\begin{array}{c}
R_{\omega, \kappa}^{\varepsilon}(-L, z) \\
1
\end{array}\right],
$$

which corresponds to the scattering problem for a unit-power incident plane wave incoming from the right half-space and probing the inhomogeneous layer in $(-L, z)$ with matched (transparent) boundary conditions (see Figure A.1). By inverting this matrix-vector relation we get

$$
T_{\omega, \kappa}^{\varepsilon}=\frac{1}{P_{22}^{\varepsilon}(\omega, \kappa,-L, z)}, \quad R_{\omega, \kappa}^{\varepsilon}=\frac{P_{12}^{\varepsilon}(\omega, \kappa,-L, z)}{P_{22}^{\varepsilon}(\omega, \kappa,-L, z)} .
$$

From the relation (A.3) with $z_{0}=-L$ and $z=0$, we get

$$
\begin{aligned}
\hat{a}^{\varepsilon}(\omega, \kappa, 0) & =\frac{P_{11}^{\varepsilon} P_{22}^{\varepsilon}(\omega, \kappa,-L, 0)-P_{12}^{\varepsilon} P_{21}^{\varepsilon}(\omega, \kappa,-L, 0)}{P_{22}^{\varepsilon}(\omega, \kappa,-L, 0)-P_{12}^{\varepsilon}(\omega, \kappa,-L, 0)} \hat{a}^{\varepsilon}\left(\omega, \kappa,(-L)^{+}\right) \\
& =\frac{\left(\operatorname{det} \mathbf{P}^{\varepsilon}(\omega, \kappa,-L, 0)\right) T_{\omega, \kappa}^{\varepsilon}(-L, 0)}{1-R_{\omega, \kappa}^{\varepsilon}(-L, 0)} \hat{a}^{\varepsilon}\left(\omega, \kappa,(-L)^{+}\right) .
\end{aligned}
$$

Since $\mathbf{M}^{\varepsilon}$ has trace zero we have $\partial_{z}\left(\operatorname{det} \mathbf{P}^{\varepsilon}\right)=\left(\operatorname{Tr} \mathbf{M}^{\varepsilon}\right)\left(\operatorname{det} \mathbf{P}^{\varepsilon}\right)=0$, and therefore $\operatorname{det} \mathbf{P}^{\varepsilon}=1$. This simplifies the expression of the transmitted right-going wave amplitude

$$
\hat{a}^{\varepsilon}(\omega, \boldsymbol{\kappa}, 0)=\frac{T_{\omega, \kappa}^{\varepsilon}}{1-R_{\omega, \kappa}^{\varepsilon}} \hat{a}^{\varepsilon}\left(\omega, \boldsymbol{\kappa},(-L)^{+}\right) .
$$

Substituting (A.2) into this expression and taking into account (A.1) gives (4.5). 


\section{REFERENCES}

[1] M. Asch, W. Kohler, G. Papanicolaou, M. Postel, and B. White, Frequency content of randomly scattered signals, SIAM Review, 33 (1991), pp. 519-626.

[2] C. Bardos, J. Garnier, and G. Papanicolaou, Identification of Green's functions singularities by cross correlation of noisy signals, Inverse Problems, 24 (2008), 015011.

[3] J. F. Claerbout, Imaging the Earth's interior, Blackwell Scientific Publications, Palo Alto, 1985.

[4] Y. Colin de Verdière, Mathematical models for passive imaging. I: general background, arXiv:math-ph/0610043v1 (2006).

[5] Y. Colin de Verdière, Mathematical models for passive imaging. II: Effective Hamiltonians associated to surface waves, arXiv:math-ph/0610044v1 (2006).

[6] A. Curtis, P. Gerstoft, H. Sato, R. Snieder, and K. Wapenaar, Seismic interferometry - turning noise into signal, The Leading Edge, 25 (2006), pp. 1082-1092.

[7] M. de Hoop and K. Sølna, Estimating a Green's function from field-field correlations in a random medium, SIAM J. Appl. Math., 69, (2009), pp. 909-932.

[8] J.-P. Fouque, J. Garnier, G. Papanicolaou, and K. Sølna, Wave propagation and time reversal in randomly layered media, Springer, New York, 2007.

[9] J. Garnier, Imaging in randomly layered media by cross-correlating noisy signals, SIAM Multiscale Model. Simul., 4 (2005), pp. 610-640.

[10] J. Garnier and G. Papanicolaou, Passive sensor imaging using cross correlations of noisy signals in a scattering medium, SIAM J. Imaging Sciences, 2 (2009), pp. 396-437.

[11] J. Garnier and K. Sølna, Wave transmission through random layering with pressure release boundary conditions, submitted, available at http://www.proba.jussieu.fr/ garnier.

[12] O. I. Lobkis and R. L. Weaver, On the emergence of the Green's function in the correlations of a diffuse field, J. Acoustic. Soc. Am., 110 (2001), pp. 3011-3017.

[13] P. Roux and M. Fink, Green's function estimation using secondary sources in a shallow water environment, J. Acoust. Soc. Am., 113 (2003), pp. 1406-1416.

[14] N. M. Shapiro, M. Campillo, L. Stehly, and M. H. Ritzwoller, High-resolution surface wave tomography from ambient noise, Science, 307 (2005), pp. 1615-1618.

[15] R. O. Schmidt, Multiple emitter location and signal parameter estimation, IEEE Trans. Antennas Propagation., 34 (1986), pp. 276280.

[16] K. Wapenaar and J. Fokkema, Green's function representations for seismic interferometry, Geophysics, 71 (2006), pp. SI33-SI46.

[17] B. White, P. Sheng, and B. Nair, Localization and backscattering spectrum of seismic waves in stratified lithology, Geophysics, 55 (1990), pp. 1158-1165.

[18] H. Yao, R. D. van der Hilst, and M. V. de Hoop, Surface-wave array tomography in SE Tibet from ambient seismic noise and two-station analysis I. Phase velocity maps, Geophysical Journal International, 166 (2006), pp. 732-744. 